\title{
Reconciling the light-cone and nonrelativistic QCD approaches to calculating $e^{+} e^{-} \rightarrow J / \psi+\eta_{c}$
}

\author{
Geoffrey T. Bodwin \\ High Energy Physics Division, Argonne National Laboratory, \\ 9700 South Cass Avenue, Argonne, Illinois 60439 \\ Daekyoung Kang and Jungil Lee* \\ Department of Physics, Korea University, Seoul 136-701, Korea
}

(Dated: August 30, 2018) 


\begin{abstract}
It has been suggested in Ref. [A. E. Bondar and V. L. Chernyak, Phys. Lett. B 612, 215 (2005)] that the disagreement between theoretical calculations and experimental observations for the rate for the process $e^{+} e^{-} \rightarrow J / \psi+\eta_{c}$ at the $B$ factories might be resolved by using the light-cone method to take into account the relative momentum of the heavy-quark and antiquark in the quarkonia. The light-cone result for the production cross section in Ref. [A. E. Bondar and V. L. Chernyak, Phys. Lett. B 612, 215 (2005)] is almost an order of magnitude larger than existing NRQCD factorization results. We investigate this apparent theoretical discrepancy. We compute light-cone distribution functions by making use of quarkonium wave functions from the Cornell potential model. Our light-cone distribution functions are similar in shape to those of Ref. [A. E. Bondar and V. L. Chernyak, Phys. Lett. B 612, 215 (2005)] and yield a similar cross section. However, when we subtract parts of the light-cone distribution functions that correspond to corrections of relative-order $\alpha_{s}$ in the NRQCD approach, we find that the cross section decreases by about a factor of three. When we set certain renormalization factors $Z_{i}$ in the light-cone calculation equal to unity, we find a further reduction in the cross section of about a factor of two. The resulting lightcone cross section is similar in magnitude to the NRQCD factorization cross sections and shows only a modest enhancement over the light-cone cross section in which the relative momentum of the heavy-quark and antiquark is neglected.
\end{abstract}

PACS numbers: 12.38.-t, 12.39.Pn, 12.38.Bx, 13.20.Gd, 14.40.Gx

*Visiting faculty, Physics Department, Ohio State University, Columbus, Ohio 43210, USA 


\section{INTRODUCTION}

One of the outstanding issues in quantum chromodynamics (QCD) in recent years is the discrepancy between the theoretical predictions and the experimental measurements for the exclusive production of $J / \psi+\eta_{c}$ in $e^{+} e^{-}$collisions at the $B$ factories. The cross section times the branching ratio into at least two charged tracks has been measured by the Belle collaboration to be $25.6 \pm 2.8 \pm 3.4 \mathrm{fb}$ (Ref. [1,2]) and by the BaBar collaboration to be $17.6 \pm 2.8_{-2.1}^{+1.5} \mathrm{fb}$ (Ref. [3]). In contrast, calculations by Braaten and Lee [4] and by Liu, He, and Chao [5] using the nonrelativistic QCD (NRQCD) factorization approach [6] at leading order in $\alpha_{s}$ yield predictions of $3.78 \pm 1.26 \mathrm{fb}$ and $5.5 \mathrm{fb}$, respectively. ${ }^{1} \mathrm{~A}$ similar disagreement between NRQCD factorization and experiment holds for production of $\chi_{c 0}$ and $\eta_{c}(2 S)$ mesons in conjunction with a $J / \psi$ meson. A recent calculation of corrections of next-to-leading order in $\alpha_{s}$ leads to an enhancement of the theoretical prediction of about a factor 1.8 (Ref. [7]). Nevertheless, a substantial discrepancy between theory and experiment remains.

It has been suggested in several recent papers [8-11] that predictions for the $J / \psi+\eta_{c}$ production cross section in the light-cone formalism might be in better agreement with experiment. In the present paper, we focus on the work of Bondar and Chernyak in Ref. [8], henceforth referred to as BC. In BC, it is claimed that an enhancement of the cross section occurs in the light-cone formalism because the expressions for the cross section contain an integration over the light-cone momentum. In contrast, in the NRQCD factorization approach, cross sections are computed as an expansion in the velocity $v$ of the heavy quark or antiquark in the quarkonium rest frame. In the leading order in $v$, the relative momentum of the heavy quark and antiquark are neglected, which is equivalent in the light-cone formalism to approximating the light-cone distribution amplitude (light-cone wave function) by a delta function at a light-cone momentum fraction of $1 / 2$. Effects of the nonzero relative momentum of the heavy quark and antiquark in NRQCD are taken into account through terms of higher order in the $v$ expansion.

The NRQCD factorization and light-cone formalisms are both believed to be valid approximations to QCD for the process $e^{+} e^{-} \rightarrow J / \psi+\eta_{c}$ in the limit in which the hard-scattering

\footnotetext{
${ }^{1}$ The differences in the predictions arise from different choices for $\alpha_{s}$ and the charmonium wave functions at the origin and from the inclusion of QED effects in the calculation of Ref. [4].
} 
momentum transfer is much greater than either the QCD scale $\Lambda_{\mathrm{QCD}}$ or the charm-quark mass $m_{c}{ }^{2}$ In NRQCD factorization, one makes the further assumption that the cross section can be written as an expansion in powers of $v$. Since the light-cone and NRQCD factorization approaches are both first-principles methods, their predictions should be in reasonable agreement unless, as is asserted in BC, the $v$ expansion of NRQCD breaks down for the process $e^{+} e^{-} \rightarrow J / \psi+\eta_{c}$.

In this paper we investigate several issues with regard to the calculation in BC by making use of light-cone distribution amplitudes that are derived from potential-model quarkonium wave functions. We first compare the light-cone distribution amplitude that we derive with the model light-cone distribution amplitudes that are used in BC. We find that they are quite similar in shape, but with significantly different asymptotic behavior at large lightcone momentum fraction, and that they lead to similar values for the cross section for $e^{+} e^{-} \rightarrow J / \psi+\eta_{c}$. We then argue that a part of the contribution to the cross section that is computed in $\mathrm{BC}$ comes from the high-momentum tail of the light-cone distribution amplitude. Such high-momentum contributions are taken into account in NRQCD through contributions to the cross section of relative order $\alpha_{s}$ and higher. Furthermore, since our potential-model wave function is accurate only for momenta that are much less than $m_{c}$, it is not legitimate for us to include the high-momentum contribution in our light-cone calculation. When we subtract this contribution, we find that the cross section is reduced by about a factor of three. A further reduction of about a factor of two occurs when we replace with unity certain renormalization factors $Z_{i}$ that appear in the calculation in BC, but which have no counterpart in a conventional NRQCD factorization calculation. With these modifications, the light-cone calculation gives a cross section that is comparable to that of the NRQCD factorization calculations.

The remainder of this paper is organized as follows. In Sec. II, we describe the Cornell potential model that we use. In Sec. III, we present the relationships of the required lightcone distribution amplitudes to the Bethe-Salpeter (BS) wave function, use the BS equation to compute the BS wave function in terms of a potential-model wave function, and give the correspondences between the terms in the resulting BS wave function and the required lightcone distribution amplitudes. Sec. IV contains the detailed expressions for the light-cone

\footnotetext{
${ }^{2}$ For a discussion of the current status of proofs of NRQCD factorization, see Refs. [12-15].
} 
distribution amplitudes in terms of the potential-model wave function and a re-arrangement of these expressions into forms that are amenable to numerical calculation. In Sec. V, we identify the high-momentum part of the light-cone distribution amplitude that is properly treated as an order- $\alpha_{s}$ correction to the cross section. We also compute and compare the asymptotic forms of the light-cone distribution amplitude and the high-momentum part in the high-momentum limit. In Sec. VI, we present the expression for the cross section in the light-cone formalism, give our numerical results for the cross section at $B$-factory energies, and discuss the implications of these results. Finally, in Sec. VII, we summarize our findings.

\section{POTENTIAL MODEL}

In this section, we describe the calculation of the potential-model wave function. We note that, if we knew the heavy-quark potential exactly, then we could calculate the heavyquarkonium wave function in a potential model, up to corrections of relative order $v^{2}$ (Ref. [16]). We make use of the Cornell potential model of Ref. [17]. The Cornell potential provides a reasonably good fit to heavy-quark potentials that are measured in lattice calculations. $^{3}$

The Schrödinger equation for the radial wave function for a quark-antiquark $(Q \bar{Q})$ pair with orbital-angular-momentum quantum number $\ell$ in a central potential $V(r)$ is

$$
\left[-\frac{1}{m r^{2}} \frac{d}{d r}\left(r^{2} \frac{d}{d r}\right)+\frac{\ell(\ell+1)}{m r^{2}}+V(r)\right] R(r)=\epsilon_{\mathrm{B}} R(r),
$$

where $\epsilon_{\mathrm{B}}$ is the binding energy, $r$ is the distance between the heavy quark and antiquark, and $m$ is mass of the heavy quark. Note that the reduced mass of the pair is $\mu=m / 2$.

The Cornell potential [17] is given by

$$
V(r)=-\frac{\kappa}{r}+\frac{r}{a^{2}}
$$

where the parameters $\kappa$ and $a$ determine the strength of Coulomb and linear potentials, respectively. For a color-singlet $Q \bar{Q}$ pair, the Coulomb-strength parameter $\kappa$ can be expressed in terms of an effective strong coupling $\alpha_{s}$ as

$$
\kappa=\alpha_{s} C_{F},
$$

\footnotetext{
${ }^{3}$ For a recent review that discusses heavy-quark potentials from lattice measurements, see Ref. [18].
} 
where $C_{F}=4 / 3$. The parameter $a$ is related to the string tension $\sigma$ as

$$
\sigma=\frac{1}{a^{2}}
$$

Following Ref. [17], we replace $\kappa$ and $r$ by dimensionless variables $\lambda$ and $\rho$ :

$$
\begin{aligned}
\kappa & =(m a)^{-2 / 3} \lambda, \\
r & =\frac{\lambda}{m \kappa} \rho=a(m a)^{-1 / 3} \rho .
\end{aligned}
$$

Substituting the Cornell potential into Eq. (1), we can rewrite the radial equation:

$$
\left[\frac{d^{2}}{d \rho^{2}}-\frac{\ell(\ell+1)}{\rho^{2}}+\frac{\lambda}{\rho}-\rho+\zeta\right] u(\rho)=0,
$$

where the dimensionless function $u(\rho)$ and dimensionless energy eigenvalue $\zeta$ in Eq. (6) are related to their physical counterparts as

$$
\begin{aligned}
R(r) & =\sqrt{\frac{m}{a^{2}}} \frac{u(\rho)}{\rho}, \\
\epsilon_{\mathrm{B}} & =m(m a)^{-4 / 3} \zeta .
\end{aligned}
$$

The wave functions are normalized according to

$$
\int_{0}^{\infty}|u(\rho)|^{2} d \rho=\int_{0}^{\infty}|R(r)|^{2} r^{2} d r=1
$$

In Eq. (2.19) of Ref. [17], the wave function at the origin is expressed in terms of the model parameters $m$ and $a$ as

$$
|\psi(0)|^{2}=\frac{m}{4 \pi a^{2}}\left(1+\lambda\left\langle\rho^{-2}\right\rangle\right)
$$

where the expectation value $\left\langle\rho^{-2}\right\rangle$ is defined by

$$
\left\langle\rho^{-2}\right\rangle=\int_{0}^{\infty} \frac{[u(\rho)]^{2}}{\rho^{2}} d \rho .
$$

Numerical values of $\left\langle\rho^{-2}\right\rangle$ for various values of $\lambda$ are given in Table I of Ref. [17]. As we explain below, we fix the value of $|\psi(0)|$ by using the leptonic decay width of the $J / \psi$. We need an additional constraint to fix both of the parameters $m$ and $a$ for a given $\lambda$. Following Ref. [17], we use the mass splitting between the $J / \psi$ and the $\psi(2 S)$. Eq. (2.12) of Ref. [17] expresses the mass splitting as

$$
M_{\psi(2 S)}-M_{J / \psi}=m(m a)^{-4 / 3}\left(\zeta_{20}-\zeta_{10}\right)
$$


where $\zeta_{n 0}$ is the eigenvalue for the radial equation (6) for the principal quantum number $n$ and the orbital-angular-momentum quantum number $\ell=0$. The values for $\zeta_{10}$ as a function of $\lambda$ are given in Table I of Ref. [17]. We use Eq. (A4) and Table II of Ref. [17] to determine $\zeta_{20}$ for various values of $\lambda$ :

$$
\zeta_{20}=4.0879-0.5826 \lambda-0.0302 \lambda^{2} .
$$

Solving Eqs. (9) and (11), we can determine the model parameters $m$ and $a$ :

$$
\begin{aligned}
m & =\frac{\zeta_{20}-\zeta_{10}}{M_{\psi(2 S)}-M_{J / \psi}}\left(\frac{4 \pi|\psi(0)|^{2}}{1+\lambda\left\langle\rho^{-2}\right\rangle}\right)^{2 / 3}, \\
a & =\left(\frac{\zeta_{20}-\zeta_{10}}{M_{\psi(2 S)}-M_{J / \psi}}\right)^{1 / 2}\left(\frac{4 \pi|\psi(0)|^{2}}{1+\lambda\left\langle\rho^{-2}\right\rangle}\right)^{-1 / 6} .
\end{aligned}
$$

From Eqs. (3) and (5a), it can be seen that the effective strong coupling $\alpha_{s}$ for the boundstate potential as a function of $\lambda$ is given by

$$
\alpha_{s}=\frac{\lambda}{C_{F}}(m a)^{-2 / 3}
$$

As we have mentioned, we determine $|\psi(0)|$ from the leptonic decay width of the $J / \psi$. NRQCD matrix elements for $J / \psi$ that correspond to $|\psi(0)|^{2}$ are given in Table I of Ref. [4]. These values are $\langle O\rangle_{S}=0.208 \mathrm{GeV}^{3}$ for $\mathrm{LO}$ and $0.335 \mathrm{GeV}^{3}$ for NLO, where the identifiers $\mathrm{LO}$ and NLO indicate that the short-distance coefficient for the leptonic decay rate of the $J / \psi$ has been computed to leading order or next-to-leading order in the strong-coupling constant. Expressing $|\psi(0)|$ in terms of these matrix elements, we obtain

$$
|\psi(0)|=\sqrt{\frac{\langle O\rangle_{J / \psi}}{2 N_{c}}}= \begin{cases}0.18619 \mathrm{GeV}^{3 / 2} & (\mathrm{LO}), \\ 0.23629 \mathrm{GeV}^{3 / 2} & \text { (NLO). }\end{cases}
$$

In this paper, we use the LO value of $|\psi(0)|$, which corresponds to the formula

$$
\Gamma\left[J / \psi \rightarrow \ell^{+} \ell^{-}\right]=\frac{4 \pi e_{c}^{2} \alpha^{2}}{m_{c}^{2}}|\psi(0)|^{2},
$$

where $e_{c}=2 / 3$ is the fractional electric charge of the charm quark. If one uses the NLO value of $|\psi(0)|$, then the average momentum-squared of the wave function is reduced by about 10\% (Ref. [19]). Therefore, we do not expect that the use of the NLO value would change our results qualitatively.

Using $|\psi(0)|=0.18619 \mathrm{GeV}^{3 / 2}, M_{J / \psi}=3.096916 \mathrm{GeV}, M_{\psi(2 S)}=3.686093 \mathrm{GeV}$, Table I of Ref. [17], and Eq. (12), we can compute $m$ and $a$ from Eq. (13). The results for various 
TABLE I: Potential-model parameters and derived quantities as a function of the strength $\lambda$ of the Coulomb potential. The definitions of the parameters and derived quantities are given in the text. The parameters are computed using the inputs $|\psi(0)|=0.18619 \mathrm{GeV}^{3 / 2}, M_{J / \psi}=3.096916 \mathrm{GeV}$, and $M_{\psi(2 S)}=3.686093 \mathrm{GeV}$, as is described in the text.

\begin{tabular}{c|lllllllll}
\hline \hline$\lambda$ & 0 & 0.2 & 0.4 & 0.6 & 0.7 & 0.8 & 1.0 & 1.2 & 1.4 \\
\hline $1+\lambda\left\langle\rho^{-2}\right\rangle$ & 1. & 1.24988 & 1.55768 & 1.93498 & 2.15369 & 2.39480 & 2.95190 & 3.62272 & 4.42524 \\
$\zeta_{10}$ & 2.33811 & 2.16732 & 1.98850 & 1.80107 & 1.70394 & 1.60441 & 1.39788 & 1.18084 & 0.95264 \\
$\zeta_{20}$ & 4.08790 & 3.97017 & 3.85003 & 3.72747 & 3.66528 & 3.60249 & 3.47510 & 3.34529 & 3.21307 \\
$m(\mathrm{GeV})$ & 1.70670 & 1.51548 & 1.35120 & 1.21003 & 1.14710 & 1.08877 & 0.98458 & 0.89501 & 0.81796 \\
$a\left(\mathrm{GeV}^{-1}\right)$ & 1.97932 & 2.08520 & 2.19805 & 2.31833 & 2.38139 & 2.44648 & 2.58295 & 2.72816 & 2.88253 \\
$\sqrt{\sigma}(\mathrm{GeV})$ & 0.50522 & 0.47957 & 0.45495 & 0.43134 & 0.41992 & 0.40875 & 0.38715 & 0.36655 & 0.34692 \\
$\sigma(\mathrm{GeV})$ & 0.25525 & 0.22999 & 0.20698 & 0.18606 & 0.17633 & 0.16708 & 0.14989 & 0.13436 & 0.12035 \\
$\alpha_{s}$ & 0. & 0.06966 & 0.14519 & 0.22624 & 0.26866 & 0.31225 & 0.40255 & 0.49634 & 0.59272 \\
$\gamma_{\mathrm{C}}(\mathrm{GeV})$ & 0. & 0.07037 & 0.13079 & 0.18250 & 0.20545 & 0.22664 & 0.26423 & 0.29615 & 0.32322 \\
\hline \hline
\end{tabular}

values of $\lambda$ are shown in Table I, along with values for $\alpha_{s}$ from Eq. (14), $\sigma$ from Eq. (4), and $\gamma_{\mathrm{C}}$, which is related to the binding energy for a pure Coulomb potential and is defined by

$$
\gamma_{\mathrm{C}}=\frac{1}{2} m \alpha_{s} C_{F}
$$

Lattice measurements of the heavy-quark potential yield values for effective coupling $\alpha_{s}$ of 0.22 in the quenched case and approximately 0.26 in the unquenched case [18]. A lattice measurement of the string tension $K=\sigma$ (Ref. [20]) gives $K a_{\mathrm{L}}^{2}=0.0114(2)$ at a lattice coupling $\beta=6.5$, where $a_{\mathrm{L}}$ is the lattice spacing. Lattice calculations of the hadron spectrum at $\beta=6.5$ yield values for $1 / a_{\mathrm{L}}$ of $3.962(127) \mathrm{GeV}$ (Refs. [21, 22]) and 3.811(59) $\mathrm{GeV}$ (Refs. [21, 23]). These yield values of the string tension of $K=0.1790 \pm 0.0119 \mathrm{GeV}^{2}$ and $K=0.1656 \pm 0.0059 \mathrm{GeV}^{2}$, respectively. Comparing the results of these lattice measurements with Table I, we conclude that $\lambda=0.7$ is a reasonable choice for the value of the potentialmodel parameter, and we use that value in our analysis.

We express the radial equation (6) as a difference equation, and integrate it numerically. The result, for the choice of parameters in Table I that corresponds to $\lambda=0.7$ is shown in 


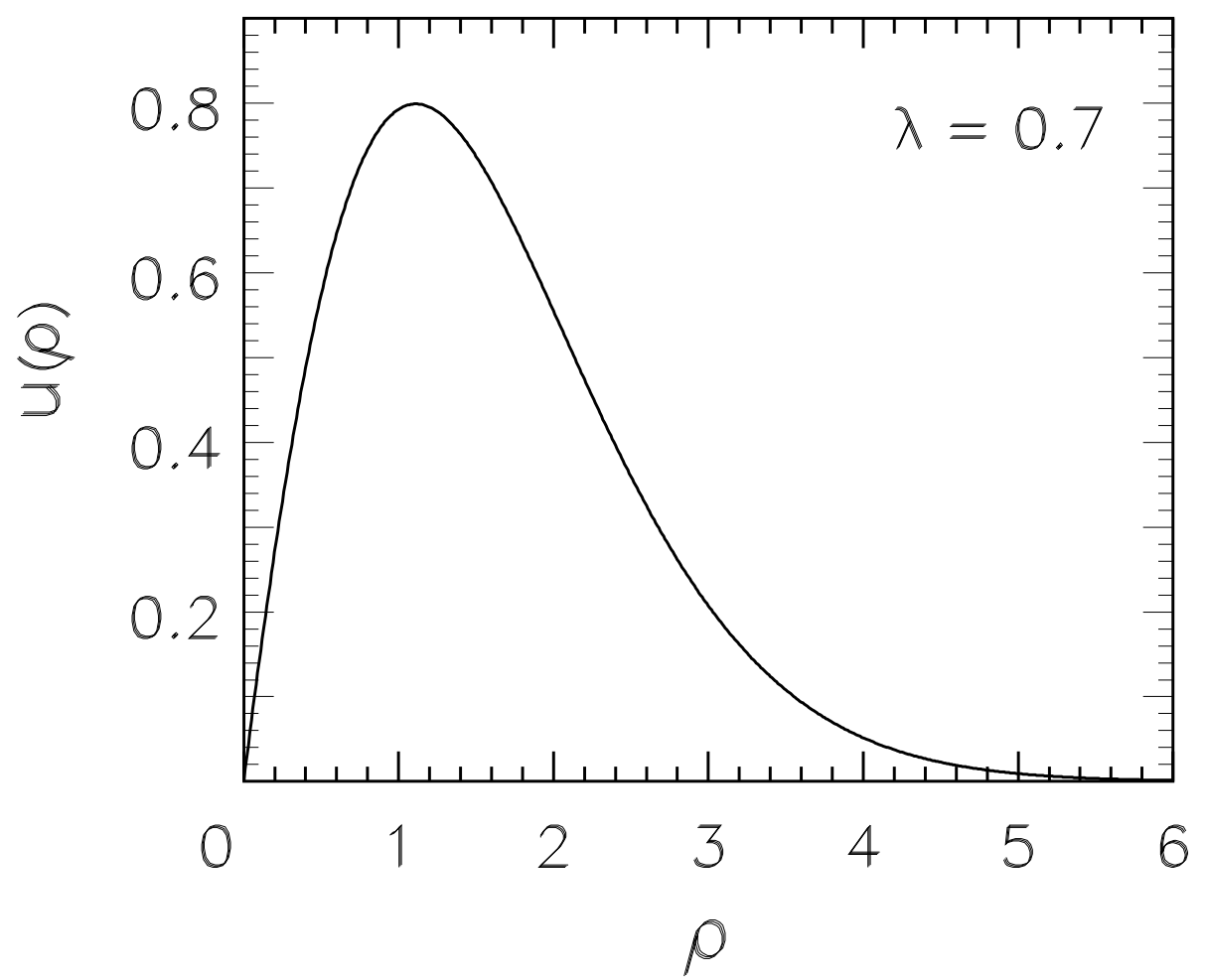

FIG. 1: The dimensionless wave function $u(\rho)$ as a function of the dimensionless variable $\rho$. Input parameters are $m=1.14710 \mathrm{GeV}, a=2.38139 \mathrm{GeV}^{-1}$, and $\lambda=0.7$. The wave function at the origin is taken to be $|\psi(0)|=0.18619 \mathrm{GeV}^{3 / 2}$, which is the value for the $J / \psi$ wave function at the origin that is designated as LO in Ref. [4].

Fig. 1.

\section{RELATIONSHIPS OF THE LIGHT-CONE DISTRIBUTION AMPLITUDES TO THE POTENTIAL-MODEL WAVE FUNCTION}

\section{A. Light-cone distribution amplitudes}

In this subsection, we re-produce the expressions that are given in $\mathrm{BC}$ for the light-cone distribution amplitudes in terms of the BS wave functions.

For the $J / \psi$ with polarization $\epsilon$, the light-cone distribution amplitudes are defined by

$$
\begin{gathered}
\left\langle J / \psi(P, \epsilon)\left|\bar{Q}_{\beta}(x) Q_{\alpha}(-x)\right| 0\right\rangle_{\mu^{*}}=\frac{f_{V} M_{J / \psi}}{4} \int_{0}^{1} d z_{1} e^{i P \cdot x\left(z_{1}-z_{2}\right)}\left\{\notin V_{\perp}(z)+\not P \frac{\epsilon \cdot x}{P \cdot x} \tilde{V}(z)\right. \\
\left.+f_{v}^{t}\left(\mu^{*}\right) \frac{\sigma_{\mu \nu} \epsilon^{\mu} P^{\nu}}{M_{J / \psi}} V_{T}(z)+f_{v}^{a}\left(\mu^{*}\right) \epsilon_{\mu \nu \rho \sigma} \gamma^{\mu} \gamma_{5} \epsilon^{\nu} P^{\rho} x^{\sigma} V_{A}(z)\right\}_{\alpha \beta}
\end{gathered}
$$


Here $Q$ and $\bar{Q}$ are the heavy quark and antiquark fields, respectively, $\mu^{*}$ is the renormalization scale, $\sigma_{\mu \nu}=\frac{1}{2}\left[\gamma_{\mu}, \gamma_{\nu}\right]$, and $z_{1}=z$ and $z_{2}=1-z$ are the fractions of the meson momentum $P^{+}=P^{0}+P^{3} \equiv q_{0}$ carried by the quark and antiquark, respectively, at large $P^{3}$. In deriving these expressions, the authors of $\mathrm{BC}$ neglect the quark transverse momentum inside the heavy quarkonium in comparison with the quark mass. This is a further approximation that goes beyond the standard light-cone formalism. As is pointed out in BC, the light-cone distribution amplitude $\widetilde{V}(z)$ may be eliminated in favor of the standard leading-twist wave function $V_{L}(z)$ of a longitudinally polarized $J / \psi$ (Ref. [24]):

$$
\left\langle J / \psi_{\lambda=0}(P)\left|\bar{Q}(x) \gamma_{\mu} Q(-x)\right| 0\right\rangle=f_{V} P_{\mu} \int_{0}^{1} d z_{1} e^{i P \cdot x\left(z_{1}-z_{2}\right)} V_{L}(z) .
$$

Taking $\epsilon$ to correspond to helicity $\lambda=0$ in Eq. (18) and using $M_{J / \psi} \epsilon_{\lambda=0}^{\mu} \rightarrow P^{\mu}$ for large $P$, one obtains

$$
V_{L}(z)=V_{\perp}(z)+\widetilde{V}(z)
$$

For the $\eta_{c}$-meson, one has

$$
\begin{gathered}
\left\langle\eta_{c}(P)\left|\bar{Q}_{\beta}(x) Q_{\alpha}(-x)\right| 0\right\rangle_{\mu^{*}}=i \frac{f_{P} M_{\eta_{c}}}{4} \int_{0}^{1} d z_{1} e^{i P \cdot x\left(z_{1}-z_{2}\right)}\left\{\frac{\not P \gamma_{5}}{M_{\eta_{c}}} P_{A}(z)-f_{p}^{p}\left(\mu^{*}\right) \gamma_{5} P_{P}(z)\right. \\
\left.+f_{p}^{t}\left(\mu^{*}\right) \sigma_{\mu \nu} P^{\mu} x^{\nu} P_{T}(z)\right\}_{\alpha \beta}
\end{gathered}
$$

\section{B. Relationships to potential-model wave functions}

In order to express the light-cone distribution amplitude in terms of a potential-model wave function, we now derive an approximate form for the BS wave function in terms of a potential-model wave function. Here we make explicit use of an expansion in the velocity $v$ of the $Q$ or $\bar{Q}$ in the quarkonium rest frame. Of course, such an expansion is not valid at large $Q \bar{Q}$ relative momenta. However, as we will discuss in detail in Sec. V, we ultimately subtract such large-momentum contributions in the cross-section calculation.

Consider a heavy $Q \bar{Q}$ pair bound in a potential $V$. The four-momenta for the pair can be written in terms of quarkonium momentum $P$ and their relative momentum $p$ as

$$
\begin{aligned}
& p_{Q}=\frac{1}{2} P+p, \\
& p_{\bar{Q}}=\frac{1}{2} P-p .
\end{aligned}
$$


In the rest frame of the heavy quarkonium, $P$ and $p$ are given by

$$
\begin{aligned}
P & =\left(M_{\text {meson }}, \mathbf{0}\right)=\left(2 m+\epsilon_{\mathrm{B}}, \mathbf{0}\right), \\
p & =\left(p^{0}, \boldsymbol{p}\right),
\end{aligned}
$$

where $\epsilon_{\mathrm{B}}$ is the binding energy of the quarkonium. Let $\psi(\boldsymbol{x})$ be the potential-model wave function satisfying the time-independent Schrödinger equation:

$$
\left(\epsilon_{\mathrm{B}}-\frac{\hat{\boldsymbol{p}}^{2}}{2 \mu}\right) \psi(\boldsymbol{x})=V(\boldsymbol{x}) \psi(\boldsymbol{x}),
$$

where $\mu=m / 2$ is the reduced mass and $\hat{\boldsymbol{p}}$ is the momentum operator. We specialize to the $S$-wave case. Then $\psi(\boldsymbol{x})=\psi(r)$, where $r=|\boldsymbol{x}|$.

We can form a trial BS wave function, accurate to leading order in $v$, by appending spin wave functions onto $\psi$. In the rest frame of the quarkonium, the spin-singlet and spin-triplet wave functions are

$$
\begin{aligned}
& \left(\Pi_{0}\right)_{a b}=\sum_{i, j} c_{i j}\left({ }^{1} S_{0}\right) \xi_{a}^{i} \bar{\eta}_{b}^{j}=\frac{1}{2 \sqrt{2}}\left[\left(1+\gamma^{0}\right) \gamma^{5}\right]_{a b}=\frac{1}{2 \sqrt{2}}\left[\left(1+\frac{\not P}{2 m}\right) \gamma^{5}\right]_{a b} \\
& \left(\Pi_{1}\right)_{a b}=\sum_{i, j} c_{i j}\left({ }^{3} S_{1}\right) \xi_{a}^{i} \bar{\eta}_{b}^{j}=-\frac{1}{2 \sqrt{2}}\left[\left(1+\gamma^{0}\right) \notin\right]_{a b}=-\frac{1}{2 \sqrt{2}}\left[\left(1+\frac{\not P}{2 m}\right) \notin\right]_{a b}
\end{aligned}
$$

where $\xi$ and $\bar{\eta}$ are Dirac spinors for a static free quark and antiquark, respectively. $c_{i j}\left({ }^{1} S_{0}\right)$ and $c_{i j}\left({ }^{3} S_{1}\right)$ are Clebsch-Gordan coefficients of the spin-singlet and spin-triplet states for the spin states $i$ and $j$ of $Q$ and $\bar{Q}$, respectively, and $a$ and $b$ are the Dirac indices. SU(3) color indices are suppressed. In the last equalities of Eqs. (25), we have written the expressions in a Lorentz-invariant form. ${ }^{4}$ Our trial wave function is then $\psi(r)\left(\Pi_{\lambda}\right)_{a b}$, where $\lambda$ takes on the values 0 or 1 . In momentum space, the trial $B S$ wave function is

$$
\widetilde{\psi}(\boldsymbol{p}) 2 \pi \delta\left(p^{0}\right)\left(\Pi_{\lambda}\right)_{a b}=\int d^{4} x e^{-i p \cdot x} \psi(r)\left(\Pi_{\lambda}\right)_{a b}
$$

The momentum-space form of the BS equation [28] is

$$
\widetilde{\Psi}_{a b}^{\mathrm{BS}}(p)=\int \frac{d^{4} q}{(2 \pi)^{4}}\left[S_{F}^{Q}(p)\right]_{a a^{\prime}}[K(p-q)]_{a^{\prime} a^{\prime \prime}, b^{\prime \prime} b^{\prime}}\left[\widetilde{\Psi}^{\mathrm{BS}}(q)\right]_{a^{\prime \prime} b^{\prime \prime}}\left[S_{F}^{\bar{Q}}(p)\right]_{b^{\prime} b},
$$

\footnotetext{
${ }^{4}$ These Lorentz-invariant expressions were first given in Refs. [25, 26]. Exact expressions, correct to all orders in $v$ were given in Ref. [27].
} 
where $S_{F}^{Q}$ is the heavy-quark propagator. In our case, we can take the interaction kernel $K$ to be

$$
[K(p-q)]_{a^{\prime} a^{\prime \prime}, b^{\prime \prime} b^{\prime}}=\gamma_{a^{\prime} a^{\prime \prime}}^{0}[-i \widetilde{V}(\boldsymbol{p}-\boldsymbol{q})] \gamma_{b^{\prime \prime} b^{\prime}}^{0}
$$

where

$$
\widetilde{V}(\boldsymbol{p}-\boldsymbol{q})=\int d^{3} x e^{i(\boldsymbol{p}-\boldsymbol{q}) \cdot \boldsymbol{x}} V(r)
$$

is the Fourier transform of the nonrelativistic potential. Then we can rewrite the BS equation as

$$
\widetilde{\Psi}_{a b}^{\mathrm{BS}}(p)=\int \frac{d^{4} q}{(2 \pi)^{4}} \frac{\left[\left(p_{Q}+m\right) \gamma^{0}\right]_{a a^{\prime}}}{p_{Q}^{2}-m^{2}+i \epsilon}[i \widetilde{V}(\boldsymbol{p}-\boldsymbol{q})] \widetilde{\Psi}_{a^{\prime} b^{\prime}}^{\mathrm{BS}}(q) \frac{\left[\gamma^{0}\left(p_{\bar{Q}}-m\right)\right]_{b^{\prime} b}}{p_{\bar{Q}}^{2}-m^{2}+i \epsilon} .
$$

Since we have neglected retardation effects, which are of relative order $v^{2}$, the interaction kernel (28) is independent of $(p-q)^{0}$. Consequently, the integration over $q^{0}$ on the right-hand side of Eq. (30) produces the equal-time BS wave function. Integrating Eq. (30) over $p^{0}$ to form the equal-time wave function on the left-hand side, we obtain the Salpeter equation [29]:

$$
\widetilde{\Psi}_{a b}^{\mathrm{S}}(\boldsymbol{p})=\int \frac{d p^{0}}{2 \pi} \int \frac{d^{3} q}{(2 \pi)^{3}} \frac{\left[\left(\not_{Q}+m\right) \gamma^{0}\right]_{a a^{\prime}}}{p_{Q}^{2}-m^{2}+i \epsilon}[i \widetilde{V}(\boldsymbol{p}-\boldsymbol{q})] \widetilde{\Psi}_{a^{\prime} b^{\prime}}^{\mathrm{S}}(\boldsymbol{q}) \frac{\left[\gamma^{0}\left(\not p_{\bar{Q}}-m\right)\right]_{b^{\prime} b}}{p_{\bar{Q}}^{2}-m^{2}+i \epsilon}
$$

where

$$
\widetilde{\Psi}_{a b}^{\mathrm{S}}(\boldsymbol{p})=\int \frac{d p^{0}}{2 \pi} \widetilde{\Psi}_{a b}^{\mathrm{BS}}(p)
$$

is the equal-time BS wave function, which is also known as the Salpeter wave function.

We can obtain a wave function that is accurate through corrections of order $v$ by substituting the trial wave function (26) into right-hand side of the Salpeter equation (31). The result is

$$
\begin{aligned}
\widetilde{\Psi}_{a b}^{\mathrm{S}}(\boldsymbol{p})=\int \frac{d p^{0}}{2 \pi} \int \frac{d^{3} q}{(2 \pi)^{3}}[i \widetilde{V}(\boldsymbol{p}-\boldsymbol{q}) \widetilde{\psi}(\boldsymbol{q})] \\
\times \frac{\left[\left(p_{Q}+m\right) \gamma^{0} \Pi_{\lambda} \gamma^{0}\left(\not p_{\bar{Q}}-m\right)\right]_{a b}}{\left(p_{Q}^{2}-m^{2}+i \epsilon\right)\left(p_{\bar{Q}}^{2}-m^{2}+i \epsilon\right)}
\end{aligned}
$$

Now we substitute Eq. (22) into Eq. (33) and carry out the the $p^{0}$ integral on the right-hand side using residue theorem. If we close the contour in the lower half-plane, then we encounter two poles:

$$
\begin{aligned}
& p^{0}=\sqrt{m^{2}+\boldsymbol{p}^{2}}-M_{\text {meson }} / 2-i \epsilon \approx \frac{1}{2}\left(\frac{\boldsymbol{p}^{2}}{m}-\epsilon_{\mathrm{B}}\right)-i \epsilon, \\
& p^{0}=\sqrt{m^{2}+\boldsymbol{p}^{2}}+M_{\text {meson }} / 2-i \epsilon \approx 2 m-i \epsilon
\end{aligned}
$$


The first pole corresponds to the positive-energy pole in the quark propagator, and the second pole corresponds to the negative-energy pole in the anti-quark propagator. The contribution from the residue at the second pole, is suppressed as $v^{2} \sim \boldsymbol{p}^{2} / m^{2} \sim\left|\epsilon_{\mathrm{B}}\right| / m$ relative to the contribution from the residue at the first pole. Evaluating the latter, we obtain

$$
\begin{aligned}
\widetilde{\Psi}_{a b}^{\mathrm{S}}(\boldsymbol{p}) \approx \int \frac{d^{3} q}{(2 \pi)^{3}}[\widetilde{V}(\boldsymbol{p}-\boldsymbol{q}) \widetilde{\psi}(\boldsymbol{q})] \\
\quad \times \frac{\left.\left[\left(p_{Q}+m\right) \gamma^{0} \Pi_{\lambda} \gamma^{0}\left(\not p_{\bar{Q}}-m\right)\right]_{a b}\right|_{p^{0}=0}}{4 m^{2}\left(\epsilon_{\mathrm{B}}-\frac{p^{2}}{2 \mu}-i \epsilon\right)},
\end{aligned}
$$

where we have neglected corrections of relative order $v^{2}$ by evaluating the numerator at $p^{0}=0$. Making use of momentum-space Schrödinger equation,

$$
\left(\epsilon_{\mathrm{B}}-\frac{\boldsymbol{p}^{2}}{2 \mu}\right) \widetilde{\psi}(\boldsymbol{p})=\int \frac{d^{3} q}{(2 \pi)^{3}} \widetilde{V}(\boldsymbol{p}-\boldsymbol{q}) \widetilde{\psi}(\boldsymbol{q}),
$$

we find that

$$
\widetilde{\Psi}_{a b}^{\mathrm{S}}(\boldsymbol{p}) \approx \frac{1}{4 m^{2}}\left[\left(p_{Q}+m\right) \gamma^{0}\right]_{a a^{\prime}}\left(\Pi_{\lambda}\right)_{a^{\prime} b^{\prime}}\left[\gamma^{0}\left(\not_{\bar{Q}}-m\right)\right]_{b^{\prime} b} \widetilde{\psi}(\boldsymbol{p}) .
$$

It is easy to see, by substituting this result into the right-hand side of the Salpeter equation (31) and repeating the preceding analysis, that this is a self-consistent solution of the Salpeter equation through terms of relative order $v$.

Finally, returning to coordinate space, we can write the equal-time BS wave functions as

$$
\begin{aligned}
\left\langle\eta_{c}(P)\left|\bar{Q} Q\left(x^{0}=0\right)\right| 0\right\rangle & \approx-\frac{1}{16 \sqrt{2} m^{3}}\left(\not p_{Q}+m\right)(\not P+2 m) \gamma^{5}\left(\not p_{\bar{Q}}-m\right) \psi(\boldsymbol{x}), \\
\left\langle J / \psi(P, \epsilon)\left|\bar{Q} Q\left(x^{0}=0\right)\right| 0\right\rangle & \approx \frac{1}{16 \sqrt{2} m^{3}}\left(\not p_{Q}+m\right)(\not P+2 m) \notin\left(\not \not_{\bar{Q}}-m\right) \psi(\boldsymbol{x}) .
\end{aligned}
$$

As we have mentioned, $p$ now has only spatial components. The interpretation of $p^{j}$ in coordinate space is $-i \nabla^{j}$. Using $P \cdot p=0$ and $P \cdot \epsilon=0$, we can rewrite the equal-time wave functions as

$$
\begin{aligned}
\left\langle\eta_{c}(P)\left|\bar{Q} Q\left(x^{0}=0\right)\right| 0\right\rangle \approx & \frac{1}{2 \sqrt{2}}\left(\gamma^{5}+\frac{1}{2 m} \not P \gamma^{5}+\frac{1}{4 m^{2}}[\not p, \not P] \gamma^{5}\right) \psi(\boldsymbol{x}), \\
\left\langle J / \psi(P, \epsilon)\left|\bar{Q} Q\left(x^{0}=0\right)\right| 0\right\rangle \approx & -\frac{1}{2 \sqrt{2}}\left(\notin+\frac{1}{2}\left[\gamma^{0}, \notin\right]+\frac{1}{2 m}\{\not p, \notin\}+\frac{i}{2 m^{2}} \epsilon_{\alpha \beta \gamma \rho} p^{\alpha} P^{\beta} \epsilon^{\gamma} \gamma^{\rho} \gamma^{5}\right) \\
& \times \psi(\boldsymbol{x}),
\end{aligned}
$$

where we have retained only terms up to those linear in $p$, and we have used

$$
\begin{aligned}
\left\{\gamma^{\alpha}, \sigma^{\mu \nu}\right\} & =-2 \epsilon^{\alpha \mu \nu \beta} \gamma_{\beta} \gamma^{5}, \\
\not p \not P \notin+\not P \notin \not p & =2 i \epsilon^{\alpha \beta \gamma \rho} p_{\alpha} P_{\beta} \epsilon_{\gamma} \gamma_{\rho} \gamma^{5} .
\end{aligned}
$$


By following a procedure similar to the one that we have just presented, one can also obtain the BS wave functions at equal light-cone time $\left(x^{+}=0\right)$. In this case, one integrates the Fourier transform of the BS equation (30) over $p^{-}$, rather than $p^{0}$. The contribution at leading order in $v$ still comes from the residue of the positive-energy pole of the heavy-quark propagator. Now, however, the mass-shell condition

$$
(P / 2+p)^{0}=\sqrt{m^{2}+\boldsymbol{p}^{2}}
$$

or, equivalently,

$$
(P / 2+p)^{-}(P / 2+p)^{+}=m^{2}+\boldsymbol{p}_{\perp}^{2}
$$

is used to eliminate $p^{-}$, rather than $p^{0}$ in the residue. Hence, one can obtain the equal-lightcone-time wave functions from the equal-time wave functions by using Eq. (41) to express $p^{3}$ in terms of $p^{+}$. We work out the details of this transformation in Sec. IV.

Comparing the definitions of the light-cone distribution amplitudes in Eqs. (18), (19), and (21) with the equal-time BS wave functions in Eqs. (39), we obtain the correspondences

$$
\begin{aligned}
P_{A} & \leftrightarrow \psi(r), \\
P_{P} & \leftrightarrow \psi(r), \\
x^{-} P_{T} & \leftrightarrow p^{-} \psi(r) \approx-p^{+} \psi(r),
\end{aligned}
$$

and

$$
\begin{aligned}
V_{\perp} & \leftrightarrow \psi(r), \\
\widetilde{V} & \leftrightarrow 0, \\
V_{T} & \leftrightarrow \psi(r), \\
x^{-} V_{A} & \leftrightarrow p^{-} \psi(r) \approx-p^{+} \psi(r) .
\end{aligned}
$$

Using Eqs. (20) and (43b), we obtain

$$
V_{L} \leftrightarrow \psi(r) .
$$

The meaning of these correspondences is that we take the Fourier transform of the quantities on the right-hand sides, use the mass-shell condition (41) to eliminate $p^{3}$ in favor of $z=$ $\left(P^{+} / 2+p^{+}\right) / P^{+}$, and integrate over $p_{\perp}$ to form the light-cone distribution at zero transverse separation. We will give the details of these steps in Sec. IV. 


\section{LIGHT-CONE DISTRIBUTION AMPLITUDES}

In this section, we compute the light-cone distribution amplitudes in terms of the potential-model wave functions, making use of the correspondences in Eqs. (42) and (43).

Let us consider first the cases in which a light-cone distribution amplitude corresponds to $\psi(r)$, as for $P_{A}, P_{P}, V_{\perp}, V_{T}$, and $V_{L}$. We begin by taking the Fourier transform of $\psi(r)$ :

$$
\widetilde{\psi}(\boldsymbol{p})=\int d^{3} x e^{i \boldsymbol{p} \cdot \boldsymbol{x}} \psi(r)
$$

From the definitions of the light-cone distribution amplitudes in Eqs. (18), (19), and (21), we see that the light-cone momentum fraction $z$ is related to the momentum variable $p$ as

$$
2 z-1=\frac{2 n \cdot p}{n \cdot P} \Rightarrow z=\frac{n \cdot(P / 2+p)}{n \cdot P}
$$

where $n=\left(0^{+}, 1^{-}, \mathbf{0}_{\perp}\right)$ is a light-like vector whose spatial component is parallel to the threemomentum $\boldsymbol{P}$ of the bound state. At the level of precision of this calculation, there is some ambiguity in the definition of $z$, in that we can discard terms of relative order $v^{2}$. Hence, we can write in the quarkonium rest frame

$$
z=\frac{P^{+} / 2+p^{+}}{P^{+}}=\frac{P^{+} / 2+p^{+}}{P^{0}} \approx \frac{P^{+} / 2+p^{+}}{P^{0}+2 p^{0}}=\frac{E_{Q}+p^{3}}{2 E_{Q}},
$$

where we have made use of the mass-shell condition (41). The quark energy $E_{Q}$ is defined as

$$
E_{Q}=\sqrt{|\boldsymbol{p}|^{2}+m_{c}^{2}}
$$

We take the last expression in Eq. (46) as our definition of $z$. This definition is identical to that which is used in $\mathrm{BC}$. It has the desirable properties that $0 \leq z \leq 1$ and that $z \leftrightarrow 1-z$ under the interchange of the quark and the antiquark. In this definition, $m_{c}$ is the pole mass of the constituent heavy quark. Note that we distinguish $m_{c}$ from the parameter $m$ that appears in the bound-state equation for our potential-model wave function. For numerical calculations in this paper we take $m_{c}=1.4 \mathrm{GeV}$.

According to the definitions of the light-cone distribution amplitudes in Eqs. (18), (19), and (21), the light-cone distribution amplitudes are given by the BS wave functions at zero transverse spatial separation, as well as zero light-cone-time separation. Therefore, in

momentum space, we obtain the light-cone distribution amplitudes by integrating over the transverse momenta of the potential-model wave functions. Carrying out this integration 
and making the change of variables to the light-cone momentum fraction $z$, we find that the light-cone distribution amplitude $\phi(z)$ is given by

$$
\phi(z)=\frac{1}{\psi(0)} \int \frac{d^{2} p_{\perp}}{(2 \pi)^{3}} \frac{\partial p^{3}}{\partial z} \widetilde{\psi}(\boldsymbol{p}),
$$

where the prefactor is introduced in order to respect the conventional normalization condition

$$
\int_{0}^{1} d z \phi(z)=1
$$

Hence, we have from Eqs. (42) and (43),

$$
P_{A}(z)=P_{P}(z)=V_{\perp}(z)=V_{T}(z)=\phi(z)
$$

Now we can simplify the expression for the light-cone distribution amplitude. The variables $p^{3}$ and $|\boldsymbol{p}|$ can be expressed in terms of $z$ and $p_{\perp} \equiv\left|\boldsymbol{p}_{\perp}\right|$ as

$$
\begin{aligned}
p^{3} & =\frac{z-\frac{1}{2}}{\sqrt{z(1-z)}} \sqrt{p_{\perp}^{2}+m_{c}^{2}}, \\
|\boldsymbol{p}| & =\sqrt{\frac{p_{\perp}^{2}+4 m_{c}^{2}\left(z-\frac{1}{2}\right)^{2}}{4 z(1-z)}} .
\end{aligned}
$$

The Jacobian in Eq. (48) is obtained from Eq. (51a) and Eq. (51b):

$$
\frac{\partial p^{3}}{\partial z}=\frac{\sqrt{p_{\perp}^{2}+m_{c}^{2}}}{4[z(1-z)]^{3 / 2}}=\frac{\sqrt{|\boldsymbol{p}|^{2}+m_{c}^{2}}}{2 z(1-z)} .
$$

The angular integral in Eq. (44) is simple to evaluate. Furthermore, for a fixed light-cone momentum fraction $z$, we can make use of Eq. (51b) to re-express the integral over the transverse momentum $\boldsymbol{p}_{\perp}$ in Eq. (48) as an integral over the $|\boldsymbol{p}|$ :

$$
\int_{-\infty}^{\infty} d^{2} p_{\perp}=8 \pi z(1-z) \int_{\sqrt{d(z)}}^{\infty} d|\boldsymbol{p}||\boldsymbol{p}|
$$

Then, the expression for the light-cone distribution amplitude reduces to

$$
\phi(z)=\frac{2}{\pi \psi(0)} \int_{\sqrt{d(z)}}^{\infty} d|\boldsymbol{p}| \sqrt{|\boldsymbol{p}|^{2}+m_{c}^{2}} \int_{0}^{\infty} d r \psi(r) r \sin (|\boldsymbol{p}| r) .
$$

The lower bound of the $|\boldsymbol{p}|$ integral is a function of $z$ :

$$
\sqrt{d(z)}=m_{c} \frac{\left|z-\frac{1}{2}\right|}{\sqrt{z(1-z)}}
$$


which is the right-hand side of Eq. (51b) at $p_{\perp}=0$. The $z$ dependence of $\phi(z)$ appears only

in the lower bound $\sqrt{d(z)}$ of the $|\boldsymbol{p}|$ integral in Eq. (54). The symmetry $\phi(z)=\phi(1-z)$ is manifest. Furthermore, it is clear that the maximum value of $\phi(z)$ occurs at $z=1 / 2$ and the minimum value (zero) occurs at $z=0$ or $z=1$.

Now let us turn to the light-cone distribution amplitudes $P_{T}$ and $V_{A}$, whose correspondences with the equal-time BS wave functions are given in Eqs. (42c) and (43d), respectively. In each of these cases, the Fourier transform replaces the factor $x^{-}$on the left side of the correspondence with a factor $\partial / \partial p^{+}$. Consequently, the solution of the resulting differential equation for $P_{T}$ or $V_{A}$ contains an integration over $p^{+}$. The expressions for $P_{T}$ and $V_{A}$ that follow from these solutions are not normalizable because this integration and the factor $p^{+}$ on the right-hand side of each correspondence lead to an ultraviolet divergence. Such ultraviolet divergences are characteristic of amplitudes involving corrections of higher order in $v$. They can be rendered finite by treating them within an effective field theory, such as NRQCD. Rather than introduce this complication into the present discussion, we simply drop such contributions to the cross section. That is, we drop the contributions that arise from the light-cone distribution amplitudes $V_{A}$ and $P_{T}$. These contributions are suppressed as $v^{2}$, and, as we shall see, they have only about a $30 \%$ affect on the cross-section calculation for the model light-cone distribution amplitudes that are used in BC. Hence, these contributions do not have a significant effect on the discussion of the order-of-magnitude discrepancy between the calculation in BC and the NRQCD factorization calculations.

\section{A. Nonrelativistic approximation}

In the nonrelativistic approximation, one neglects the relative momenta of the heavy quarks in comparison with $m_{c}$. In the light-cone formalism, this amounts to taking $\boldsymbol{p}_{\perp}=\mathbf{0}_{\perp}$ and $z=1 / 2$. In this approximation, the Jacobian in Eq. (52) reduces to

$$
\left[\frac{\partial p^{3}}{\partial z}\right]_{\text {approx }}=2 m_{c} .
$$

The corresponding approximate version of Eq. (54) is

$$
\phi_{\text {approx }}(z)=z(1-z) \frac{8 m_{c}}{\pi \psi(0)} \int_{0}^{\infty} d r \psi(r) \cos [\sqrt{d(z)} r]
$$

The approximate Jacobian (56) was used in BC. For a given potential-model wave function, it leads to a narrower light-cone distribution amplitude than does the exact Jacobian (52). 
Since, in this paper, we are generally working at leading order in $v$, it is unnecessary (but not inconsistent) to include the corrections of higher order in $v$ that are contained in the exact Jacobian. However, it is convenient to use the exact Jacobian because it preserves the normalizations of the light-cone distributions, and we have used it to derive all of the numerical results that are presented in this paper. As a check, we have also carried out numerical calculations using the approximate Jacobian (56), and those calculations are qualitatively consistent with the conclusions that we draw from the calculations that are based on the exact Jacobian.

\section{B. Expressions for the light-cone distribution amplitudes for efficient numerical} evaluation

The double integral in Eq. (54) is difficult to evaluate numerically because of the oscillatory nature of the inner integrand and because the integrand of the outer integral decreases only slowly as $|\boldsymbol{p}| \rightarrow \infty$. We can improve the numerical accuracy of the integrations by splitting the integral into two pieces:

$$
\phi(z)=\phi_{a}(z)+\phi_{b}(z)
$$

where

$$
\begin{aligned}
& \phi_{a}(z)=\frac{2}{\pi \psi(0)} \int_{\sqrt{d(z)}}^{\infty} d|\boldsymbol{p}||\boldsymbol{p}| \int_{0}^{\infty} d r \psi(r) r \sin (|\boldsymbol{p}| r) \\
& \phi_{b}(z)=\frac{2}{\pi \psi(0)} \int_{\sqrt{d(z)}}^{\infty} d|\boldsymbol{p}|\left(\sqrt{|\boldsymbol{p}|^{2}+m_{c}^{2}}-|\boldsymbol{p}|\right) \int_{0}^{\infty} d r \psi(r) r \sin (|\boldsymbol{p}| r) .
\end{aligned}
$$

The integrand in $\phi_{a}(z)$ contains the leading behavior of the integrand in Eq. (54), but is a simpler form in which the $|\boldsymbol{p}|$ integration can be carried out analytically. The integral $\phi_{b}(x)$ contains the remainder, whose integrand converges more rapidly as $|\boldsymbol{p}| \rightarrow \infty$. We carry out the integration over $|\boldsymbol{p}|$ in $\phi_{a}(z)$, treating the divergent integration as a Fourier transform whose result is defined in the distribution sense. The result is

$$
\phi_{a}(z)=1+\frac{2}{\pi \psi(0)} \int_{0}^{\infty} d r \psi(r)\left\{\sqrt{d(z)} \cos [\sqrt{d(z)} r]-\frac{\sin [\sqrt{d(z)} r]}{r}\right\} .
$$

We use Eqs. (59b) and (60) to evaluate $\phi(z)$ numerically. 
It is useful, in checking the accuracy of numerical-integration methods that we use, to know the values of the light-cone distribution amplitudes for special cases in which they can be computed analytically. The light-cone distribution amplitudes $\phi, \phi_{a}$, and $\phi_{b}$ are computed for the case of a pure Coulomb potential in Appendix A. Their asymptotic behaviors at $z=0$ (or $z=1$ ) are also given. In addition, it is simple to compute the value of $\phi_{a}$ at $z=1 / 2$. In this case $d(z)=0$, and Eq. (60) reduces to

$$
\phi_{a}(1 / 2)=1
$$

\section{Light-cone distribution amplitudes}

In Fig. 2, we show the light-cone distribution amplitude that results from substituting our potential-model wave function into Eqs. (59b) and (60) and carrying out the integrations numerically, taking $m_{c}=1.4 \mathrm{GeV}$. For comparison, we also show the model light-cone distribution amplitude that is used in $\mathrm{BC}$ :

$$
\phi_{\mathrm{BC}}(z)=c\left(v^{2}\right) z(1-z)\left[\frac{z(1-z)}{1-4 z(1-z)\left(1-v^{2}\right)}\right]^{1-v^{2}},
$$

where $v^{2}=0.3$ and $c(0.3) \approx 9.62$. As can be seen, the model light-cone distribution amplitude of $\mathrm{BC}$ is very similar in shape to the light-cone distribution amplitude that we have derived from a potential model. However, there is a significant difference in the functional forms in the tails at $z=0,1$. The asymptotic behavior of the $\mathrm{BC}$ light-cone distribution

amplitude at $z=0$ is $\phi_{\mathrm{BC}}(z) \sim c\left(v^{2}\right) z^{\left(2-v^{2}\right)}$. In contrast, the potential-model light-cone distribution amplitude that we derive behaves at $z=0$ as $\phi(z) \sim 8 \gamma_{C} z^{1 / 2} /\left(\pi m_{c}\right)$ [Eq. (A8)].

In Fig. 3, we show the light-cone distribution amplitude $\phi_{\text {approx }}(z)$ that arises from Eq. (57), in which the nonrelativistic approximation has been used for the light-cone-fraction Jacobian. As can be seen from the figure, this approximation leads to a narrower light-cone distribution than does the exact expression.

\section{PERTURBATIVE CORRECTION CONTAINED IN THE LIGHT-CONE DIS- TRIBUTION AMPLITUDE}

As can be seen from Eq. (51b), when $\phi(z)$ is used in computing the cross section, it yields contributions that arise from both large and small $Q \bar{Q}$ relative momentum $p$. The 


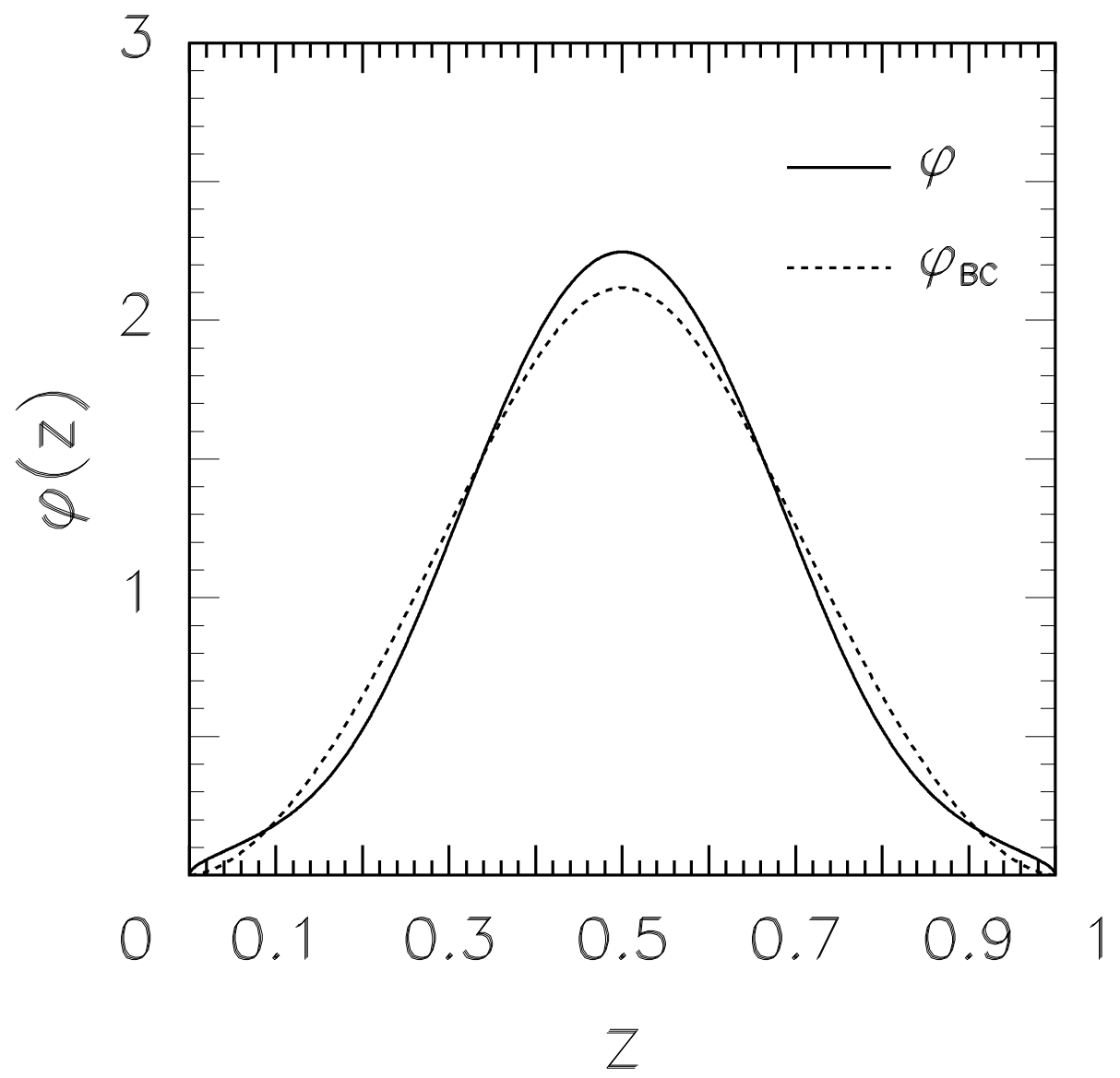

FIG. 2: The light-cone distribution amplitude $\phi(z)$ that is derived from the potential model in this paper and the model light-cone distribution amplitude $\phi_{\mathrm{BC}}(z)$ that is used in $\mathrm{BC}$. As is explained in the text, $\phi(z)$ is computed from the potential-model wave function by carrying out the integrations in Eqs. (59b) and (60) numerically, taking $m_{c}=1.4 \mathrm{GeV} . \phi_{\mathrm{BC}}(z)$ is given in Eq. (62).

large- $p$ contributions are not treated correctly in a potential model, which is inherently nonrelativistic. In the NRQCD factorization approach, this difficulty is dealt with by factoring the large- $p$ contributions into short-distance coefficients and the small- $p$ contributions into NRQCD matrix elements. The large- $p$ contributions are then computed in perturbation theory, which is presumed to be valid, owing to asymptotic freedom.

In the light-cone calculation, we also wish to identify, and ultimately remove, these large$p$ contributions. In particular, we wish to remove that part of the large- $p$ contribution that, in the NRQCD approach, is computed as an order- $\alpha_{s}$ contribution to the cross section, i.e., an order- $\alpha_{s}$ contribution to the short-distance coefficient. Of course, we could, in principle, remove contributions of order $\alpha_{s}^{2}$ and higher, as well. These contributions, presumably, are 


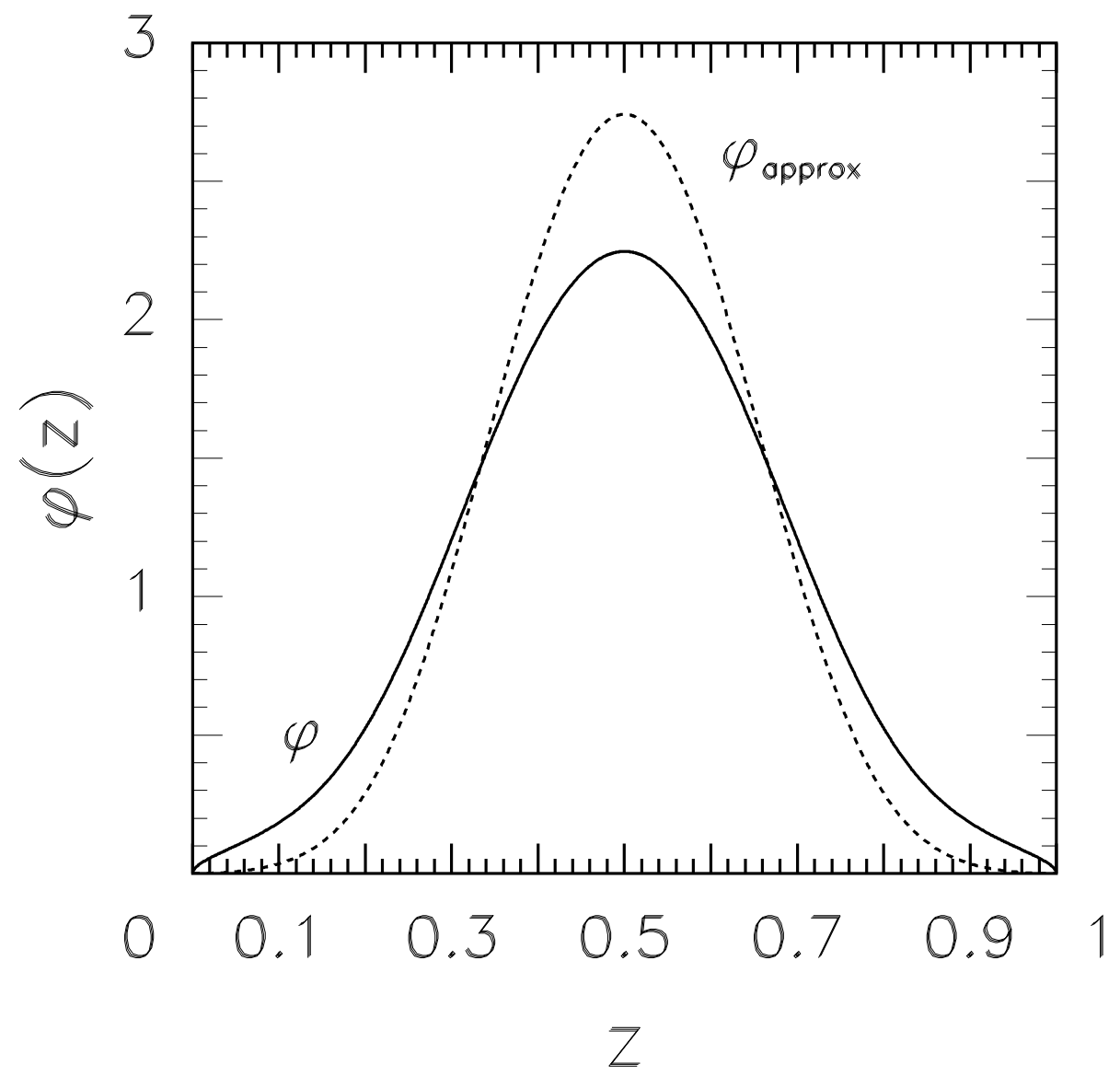

FIG. 3: Comparison of the light-cone distribution amplitude $\phi_{\text {approx }}(z)$ with $\phi(z)$. $\phi_{\text {approx }}(z)$ is computed from Eq. (57), in which the nonrelativistic approximation is used for the light-conefraction Jacobian. $\phi(z)$ is computed from the exact expressions in Eqs. (59b) and (60).

less important than the order- $\alpha_{s}$ contribution.

We begin by writing the production amplitude for the heavy-quarkonium in a schematic form, which is depicted in Fig. 4:

$$
\mathcal{M}=\int \frac{d^{4} p}{(2 \pi)^{4}} 2 \pi \delta\left(p^{0}\right) \widetilde{\psi}(\boldsymbol{p}) H(p),
$$

where $\widetilde{\psi}(\boldsymbol{p})$ is the spatial part of the momentum-space wave function for the meson and $H(p)$ is the short-distance production amplitude for the quark pair. Here we approximate the temporal part of the wave function with $2 \pi \delta\left(p^{0}\right)$. That is, we neglect deviations of the quark energy from $m$, which are of relative order $v^{2}$. In the rest frame of the meson, $\boldsymbol{p}$ is the momentum of the charm quark and $-\boldsymbol{p}$ is that for the antiquark. At this point, we could simply impose a cutoff on the integration over $\boldsymbol{p}$ in order to separate the largeand small-p contributions. However, we wish to maintain consistency with dimensional 


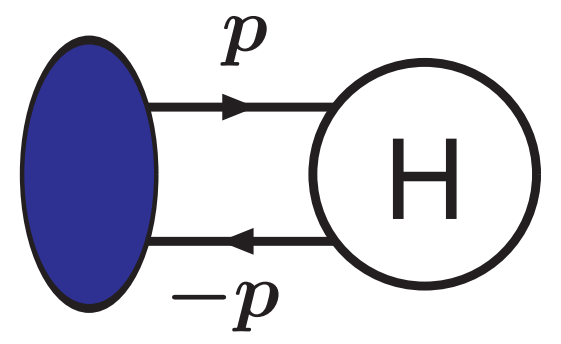

FIG. 4: Diagrammatic representation of $\mathcal{M}$, as given in Eq. (63). The circle labeled $H$ represents the hard part of the production amplitude, and the oval represents the quarkonium wave function.

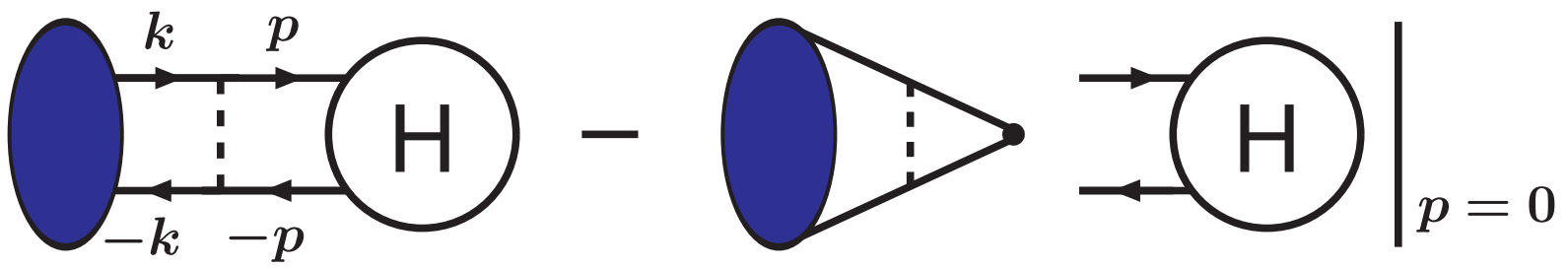

FIG. 5: Diagrammatic representation of $\mathcal{M}-\mathcal{M}_{0}$, as given in Eq. (65). The circle labeled $H$ represents the hard part of the production amplitude, and the oval represents the quarkonium wave function.

regularization, which is conventionally used to compute the short-distance coefficients in perturbation theory in the NRQCD method. Therefore, we use the method of regions [30] to compute the large and small- $p$ contributions. For small $p$, we approximate $H(p)$ by $H(0)$. Then, we obtain the standard leading-order contribution to the cross section:

$$
\mathcal{M}_{0}=\int \frac{d^{3} p}{(2 \pi)^{3}} \widetilde{\psi}(\boldsymbol{p}) H(0)=\psi(0) H(0) .
$$

We subtract $\mathcal{M}_{0}$ from $\mathcal{M}$. This removes a scaleless power infrared divergence, which vanishes in dimensional regularization. The remainder is infrared finite, and it is dominated by large $p$. Therefore, we can use the Bethe-Salpeter equation with the Coulomb-exchange kernel to write $\widetilde{\psi}(\boldsymbol{p})$ approximately in a form in which one Coulomb loop is exposed. The result, which is depicted in Fig. 5, is

$$
\begin{aligned}
\mathcal{M}-\mathcal{M}_{0}= & \int \frac{d^{4} k}{(2 \pi)^{4}} 2 \pi \delta\left(k^{0}\right) \widetilde{\psi}(\boldsymbol{k}) \int \frac{d p^{0} d^{3} p}{(2 \pi)^{4}}\left(-i g_{s} T^{a}\right)\left(+i g_{s} T^{a}\right) \frac{i}{(\boldsymbol{p}-\boldsymbol{k})^{2}} \frac{i}{p^{0}-\frac{\boldsymbol{p}^{2}}{2 m}+i \epsilon} \\
& \times \frac{-i}{p^{0}+\frac{\boldsymbol{p}^{2}}{2 m}-i \epsilon}[H(p)-H(0)],
\end{aligned}
$$


where $g_{s}$ is the strong coupling, and $T^{a}$ is an $\mathrm{SU}(3)$ color matrix. Note that we use the nonrelativistic expressions for the $Q$ and $\bar{Q}$ propagators because we wish to identify the contribution to the high-momentum tail of the light-cone distribution that arises in the potential model. In the potential model, the heavy quark and antiquark are treated in the leading nonrelativistic approximation. Taking the small- $k$ approximation for the integrand of the $\boldsymbol{k}$ integration, we obtain the order- $\alpha_{s}$ short-distance contribution to $\mathcal{M}$ :

$$
\Delta \mathcal{M}=\psi(0) \int \frac{d p^{0} d^{3} p}{(2 \pi)^{4}}\left(-i g_{s} T^{a}\right)\left(+i g_{s} T^{a}\right) \frac{i}{p^{2}-i \epsilon} \frac{i}{p^{0}-\frac{p^{2}}{2 m}+i \epsilon} \frac{-i}{p^{0}+\frac{p^{2}}{2 m}-i \epsilon}[H(p)-H(0)] .
$$

We emphasize that $\Delta \mathcal{M}$ is not arbitrary, but has a precise definition in NRQCD: $\Delta \mathcal{M} / \psi(0)$ is the order- $\alpha_{s}$ short-distance coefficient of the NRQCD operator $\psi \chi^{\dagger}$ in a QCDlike theory in which the $Q$ and $\bar{Q}$ are treated in the leading nonrelativistic approximations and the only interactions are Coulomb-gluon ladder exchanges. That theory is, of course, identical to the potential model. $\Delta \mathcal{M} / \psi(0)$ is equal to the diagrams in Fig. 5, with the wave function truncated and $k$ set to zero. That equality is precisely the NRQCD matching condition: The first diagram in Fig. 5 is the order- $\alpha_{s}$ correction to the on-shell hard-scattering in the full theory, and the second diagram in Fig. 5 is the order- $\alpha_{s}$ correction to the NRQCD operator times the leading-order short-distance coefficient. (We assume that the on-shell matrix element of $\psi \chi^{\dagger}$, which multiplies $\Delta \mathcal{M} / \psi(0)$, is normalized to unity.)

The integral over $p^{0}$ in Eq. (66) can be carried out by the contour method. Here, we assume that any singularities in $H(p)$ are of order $m$ away from the origin and, therefore correspond to contributions that are suppressed as $\boldsymbol{p}^{2} / \mathrm{m}^{2} \sim v^{2}$ relative to the leading contributions, which come from the poles at $p^{0}= \pm \boldsymbol{p}^{2} /(2 m)$. The result is then

$$
\begin{aligned}
\Delta \mathcal{M} & =4 \pi \alpha_{s} C_{F} \psi(0) \int \frac{d^{3} p}{(2 \pi)^{3}} \frac{m}{\boldsymbol{p}^{4}}[H(\boldsymbol{p})-H(\mathbf{0})] \\
& =4 \pi \alpha_{s} C_{F} \psi(0) \int \frac{d^{3} p}{(2 \pi)^{3}} \frac{m}{\left[\boldsymbol{p}^{4}\right]_{+\boldsymbol{p}}} H(\boldsymbol{p}),
\end{aligned}
$$

where

$$
H(\boldsymbol{p})=\left.H(p)\right|_{p^{0}=0} .
$$

Here, we have neglected deviations of $p^{0}$ from 0 in $H(p)$, with errors of relative order $v^{2}$. The second equality in Eq. (67a), defines the + distribution with respect to $\boldsymbol{p}$. We use the + distribution to write $\Delta \mathcal{M}$ as a convolution of the correction to the momentum-space wave 
function $\Delta \widetilde{\psi}(\boldsymbol{p})$ with the hard-scattering amplitude $H(\boldsymbol{p})$ :

$$
\Delta \mathcal{M}=\int \frac{d^{3} p}{(2 \pi)^{3}} \Delta \widetilde{\psi}(\boldsymbol{p}) H(\boldsymbol{p}),
$$

where

$$
\Delta \widetilde{\psi}(\boldsymbol{p})=4 \pi \alpha_{s} C_{F} \psi(0) \frac{m}{\left[\boldsymbol{p}^{4}\right]_{+\boldsymbol{p}}} .
$$

Therefore, we can remove contributions to the production amplitude that correspond to the standard order- $\alpha_{s}$ corrections to the NRQCD short-distance coefficient by modifying the momentum-space wave function according to $\widetilde{\psi}(\boldsymbol{p}) \rightarrow \widetilde{\psi}(\boldsymbol{p})-\Delta \widetilde{\psi}(\boldsymbol{p})$.

Similarly, we modify the light-cone distribution amplitude $\phi(z)$ according to $\phi(z) \rightarrow$ $\phi(z)-\Delta \phi(z)$. The definition of $\Delta \phi(z)$ follows from the definition of $\phi(z)$ in Eq. (48):

$$
\begin{aligned}
\Delta \phi(z) & =\frac{1}{\psi(0)} \int \frac{d^{2} p_{\perp}}{(2 \pi)^{3}} \frac{\partial p^{3}}{\partial z} \Delta \widetilde{\psi}(\boldsymbol{p}) \\
& =4 \pi m \alpha_{s} C_{F} \int \frac{d^{2} p_{\perp}}{(2 \pi)^{3}}\left[\frac{\partial p^{3}}{\partial z} \frac{1}{\boldsymbol{p}^{4}}\right]_{+z},
\end{aligned}
$$

The + distribution with respect to $z$ in the last line of Eq. (69) is defined by

$$
\int_{0}^{1} d z[f(z)]_{+z} H(z)=\int_{0}^{1} d z f(z)[H(z)-H(1 / 2)] .
$$

The evaluation of $\Delta \phi(z)$ can be carried out in analogy with the derivation of Eq. (54) from Eq. (48). The exact Jacobian in Eq. (52) is substituted into Eq. (69) and the $\boldsymbol{p}_{\perp}$ integral can be written as an integral over $|\boldsymbol{p}|$, as in Eq. (53). Then, the expression for $\Delta \phi(z)$ can be written as a single integral:

$$
\Delta \phi(z)=\frac{2 m \alpha_{s} C_{F}}{\pi} \int_{\sqrt{d(z)}}^{\infty} d|\boldsymbol{p}|\left[\frac{\sqrt{|\boldsymbol{p}|^{2}+m_{c}^{2}}}{p^{3}}\right]_{+z} .
$$

Using the identity

$$
\int \frac{\sqrt{x^{2}+1}}{x^{3}} d x=-\left[\frac{\sqrt{x^{2}+1}}{2 x^{2}}+\frac{1}{4} \log \frac{\sqrt{x^{2}+1}+1}{\sqrt{x^{2}+1}-1}\right],
$$

we obtain an analytic expression for $\Delta \phi(z)$ :

$$
\Delta \phi(z)=\frac{2 \alpha_{s} C_{F}}{\pi}\left(\frac{m}{m_{c}}\right)\left\{\left[\frac{\sqrt{z(1-z)}}{4\left(z-\frac{1}{2}\right)^{2}}\right]_{+z}+\frac{1}{4}\left[\log \frac{1+2 \sqrt{z(1-z)}}{1-2 \sqrt{z(1-z)}}\right]_{+z}\right\} .
$$

We note that, because of the definition of $\Delta \phi$ as a + distribution, $\Delta \phi$ has the property

$$
\int_{0}^{1} d z \Delta \phi(z)=0
$$


That is, the effect of subtracting $\Delta \phi$ from $\phi$ is to shift contributions from $z$ near 0 or 1 to $z$ near $1 / 2$ without changing the overall normalization of the light-cone distribution amplitude.

As can be seen from Eq. (73), $\Delta \phi$ diverges near $z=1 / 2$ as $1 /(z-1 / 2)^{2}$. When $\Delta \phi$ is multiplied by a function $f(z)$ and integrated over $z$, the leading linear divergence at $z=1 / 2$ is canceled by the factor $f(z)-f(1 / 2)$ that appears in the integrand by virtue of the definition of the + distribution. The subleading logarithmic divergence cancels because the corresponding integrand is odd about the point $z=1 / 2$.

Since the Coulomb potential controls the behavior of the wave function at large momenta (short distances), we expect the asymptotic behavior of $\Delta \phi(z)$ near $z=0$ (or $z=1$ ) to be identical to that of $\phi(z)$. It is easy to see from Eq. (73), that the asymptotic behavior of $\Delta \phi(z)$ near $z=0$ is

$$
\Delta \phi(z) \sim \frac{8 \gamma_{\mathrm{C}}}{\pi m_{c}} \sqrt{z}
$$

where we have made use of Eq. (17). (The asymptotic behavior near $z=1$ can be obtained from Eq. (75) by replacing $z$ with $1-z$.) As expected, the asymptotic behavior of $\Delta \phi(z)$ in Eq. (75) is identical to that of $\phi(z)$ in Eq. (A8a).

In Figs. 6 and 7, we plot $\Delta \phi(z)$, along with $\phi(z)$. Since $\Delta \phi$ is a + distribution [Eq. (73)], it contains a large negative contribution at $z=1 / 2$ that is not shown in Fig. 6. As can be seen from Figs. 6 and $7, \Delta \phi(z)$ shows the expected approach to $\phi(z)$ at $z=0$ and $z=1$. In Fig. 7, we show this behavior in more detail near $z=0$. The convergence of $\phi(z)$ and $\Delta \phi(z)$ at the end points has a significant consequence in the evaluation of the cross section. As we have mentioned, we must subtract $\Delta \phi(z)$ from $\phi(z)$ in order to remove contributions that are conventionally calculated as part of the order- $\alpha_{s}$ contributions to the NRQCD shortdistance coefficient. As we shall see, the cancellation between $\phi(z)$ and $\Delta \phi(z)$ at the end points results in great reduction in the cross section.

\section{LIGHT-CONE CROSS SECTION FOR $e^{+} e^{-} \rightarrow J / \psi+\eta_{c}$ AT $B$ FACTORIES}

In this section, we use the light-cone distribution amplitude that we have derived from the potential model to compute the cross section for $e^{+} e^{-} \rightarrow J / \psi+\eta_{c}$ at the $B$ factories. 


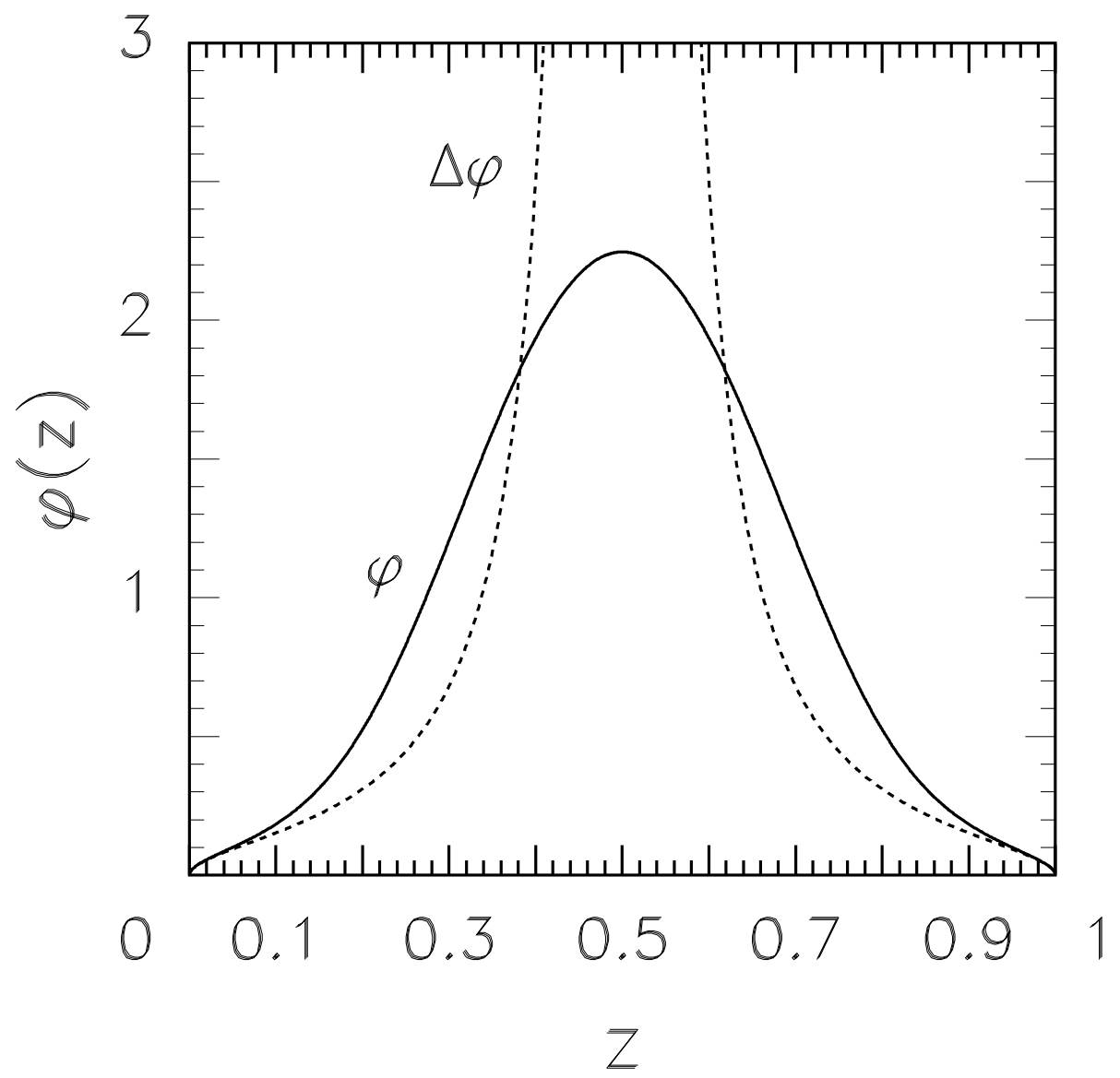

FIG. 6: $\Delta \phi(z)$ and $\phi(z)$, plotted over the full range of $z . \Delta \phi(z)$ is given by Eq. (73) and $\phi(z)$ is computed from the potential-model wave function by carrying out the integrations in Eqs. (59b) and (60) numerically. Note that $\Delta \phi$ is actually a + distribution, as is described in the text, and, therefore, it contains a large negative contribution at $z=1 / 2$ that is not shown in the figure.

\section{A. Light-cone cross section}

We begin by recording the expression for the light-cone cross section from BC. In order to compare with the results of $\mathrm{BC}$, we have tried to make use of the same approximations that were employed in that paper. (In some cases, it was necessary to deduce the approximations that were taken in $\mathrm{BC}$ by exploring the effects of various approximations on the numerical results.) In this section, we make use of the notation of BC. In particular, we use $\bar{M}_{Q}$ to represent the heavy-quark mass, and we distinguish its numerical value from those of the parameter $m$ in the potential model and the charm-quark pole mass $m_{c}$.

The light-cone cross section is derived by standard techniques $[24,31]$ and is given in $\mathrm{BC}$ 


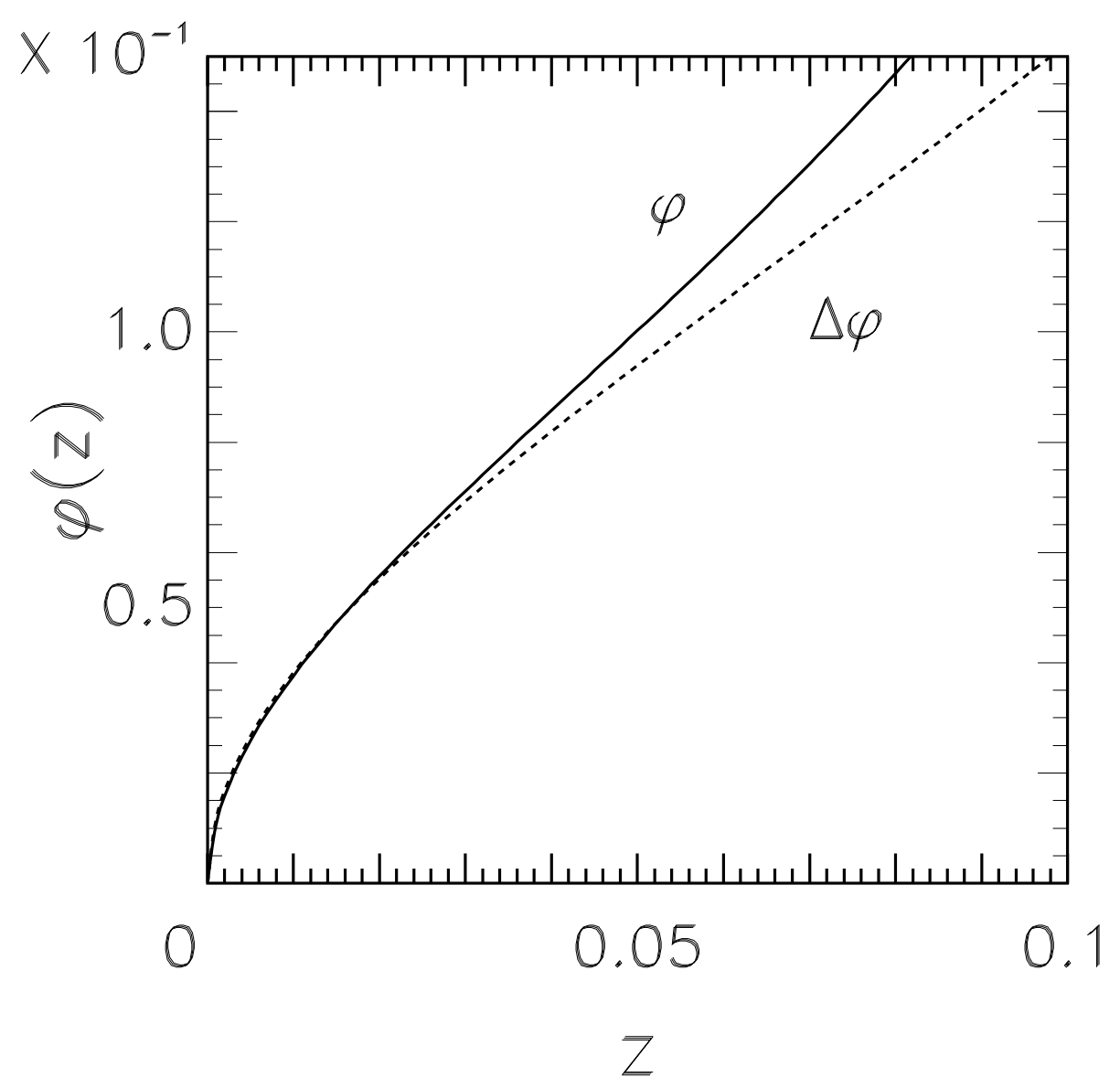

FIG. 7: $\Delta \phi(z)$ and $\phi(z)$, plotted over a restricted range in $z . \Delta \phi(z)$ is given by Eq. (73) and $\phi(z)$ is computed from the potential-model wave function by carrying out the integrations in Eqs. (59b) and (60) numerically.

as

$$
\sigma\left(e^{+} e^{-} \rightarrow J / \psi+\eta_{c}\right)=\frac{\pi \alpha^{2} e_{c}^{2}}{6}\left(\frac{|\boldsymbol{p}|}{E_{\text {beam }}}\right)^{3}\left|F_{V P}(s)\right|^{2},
$$

where $\boldsymbol{p}$ is the three momentum of the $J / \psi$ in the $e^{+} e^{-}$center-of-momentum (CM) frame and $\sqrt{s}=2 E_{\text {beam }}$ is the $e^{+} e^{-}$CM energy. The form factor $F_{V P}(s)$ is given by

$$
\left|F_{V P}(s)\right|=\frac{32 \pi}{9}\left|\frac{f_{V} f_{P} \bar{M}}{q_{0}^{4}}\right| I_{0},
$$

where $\bar{M}=\left(3 M_{J / \psi}+M_{\eta_{c}}\right) / 4$ is spin-averaged $S$-wave charmonium mass and $f_{V}$ and $f_{P}$ are the decay constants of the $J / \psi$ and the $\eta_{c}$, respectively. The quantities $|\boldsymbol{p}|$ and $q_{0}^{2}$ are given by

$$
\begin{aligned}
|\boldsymbol{p}| & =\sqrt{E_{\text {beam }}^{2}-\bar{M}^{2}}, \\
q_{0}^{2} & =\left(E_{\text {beam }}+|\boldsymbol{p}|\right)^{2} .
\end{aligned}
$$


$I_{0}$ in Eq. (77) is defined by

$$
I_{0}=\int_{0}^{1} d x_{1} \int_{0}^{1} d y_{1} \alpha_{s}\left(k^{2}\right) \sum_{i=1}^{5} f_{i}(x, y),
$$

where $k$ is the momentum of the virtual gluon. The labeling of the light-cone momentum fractions for the $J / \psi, x_{1}, x_{2}=1-x_{1}$, and for the $\eta_{c}, y_{1}, y_{2}=1-y_{1}$, can be read from Fig. 1 of BC. The integrands $f_{i}(x, y)$ in Eq. (79) are given by

$$
\begin{aligned}
& f_{1}(x, y)=\frac{Z_{t} Z_{p} V_{T}(x) P_{P}(y)}{d(x, y) s(x)} \\
& f_{2}(x, y)=-\frac{\bar{M}_{Q}^{2}}{\bar{M}^{2}} \frac{Z_{m}^{\sigma} Z_{t} V_{T}(x) P_{A}(y)}{d(x, y) s(x)} \\
& f_{3}(x, y)=\frac{1}{2} \frac{V_{L}(x) P_{A}(y)}{d(x, y)} \\
& f_{4}(x, y)=\frac{1}{2} \frac{\left(1-2 y_{1}\right)}{s(y)} \frac{V_{\perp}(x) P_{A}(y)}{d(x, y)} \\
& f_{5}(x, y)=\frac{1}{8}\left(1-Z_{t} Z_{m}^{k} \frac{4 \bar{M}_{Q}^{2}}{\bar{M}^{2}}\right) \frac{\left(1+y_{1}\right) V_{A}(x) P_{A}(y)}{d^{2}(x, y)} .
\end{aligned}
$$

The function $d(x, y)$ in Eq. (80) originates from the gluon propagator in Fig. 1 of BC and is defined by

$$
\begin{aligned}
d(x, y) & =\frac{k^{2}}{q_{0}^{2}}=\left(x_{1}+\frac{\delta}{y_{1}}\right)\left(y_{1}+\frac{\delta}{x_{1}}\right) \\
\delta & =\left(Z_{m}^{k} \frac{\bar{M}_{Q}}{q_{0}}\right)^{2} .
\end{aligned}
$$

The functions $s(x)$ and $s(y)$ in Eq. (80) are from the charm-quark propagator in Fig. 1 of $\mathrm{BC}$ and are defined by

$$
\begin{aligned}
& s(x)=x_{1}+\frac{Z_{m}^{\sigma} \bar{M}_{Q}^{2}}{y_{1} y_{2} q_{0}^{2}}, \\
& s(y)=y_{1}+\frac{Z_{m}^{\sigma} \bar{M}_{Q}^{2}}{x_{1} x_{2} q_{0}^{2}} .
\end{aligned}
$$

One might suppose that these expressions should contain two factors of $Z_{m}^{k}$ for each factor of $\bar{M}_{Q}^{2}$, as is the case in the corresponding expressions in Refs. [10, 11]. Here, we wish to compare directly with the results of $\mathrm{BC}$, and so we use the expressions that are given in that paper. The inclusion of two factors of $Z_{m}^{k}$ for each factor of $\bar{M}_{Q}^{2}$ in Eq. (82) has a small numerical effect on the cross section, which we will describe in Sec. VIC. Following BC, we 
also take

$$
\begin{aligned}
E_{\text {beam }} & =\frac{\sqrt{s}}{2} \\
|\boldsymbol{p}| & \approx E_{\text {beam }}-\frac{\bar{M}^{2}}{2 E_{\text {beam }}} \\
q_{0} & \approx \sqrt{s-2 \bar{M}^{2}} \\
k^{2} & \approx x_{1} y_{1} q_{0}^{2}+2 \bar{M}_{Q}^{2}
\end{aligned}
$$

The various renormalization factors that appear in Eqs. (80) and (82) are defined by

$$
\begin{aligned}
Z_{p} & =\left[\frac{\alpha_{s}\left(k^{2}\right)}{\alpha_{s}\left(\bar{M}_{Q}^{2}\right)}\right]^{-3 C_{F} / b_{0}}, \\
Z_{t} & =\left[\frac{\alpha_{s}\left(k^{2}\right)}{\alpha_{s}\left(\bar{M}_{Q}^{2}\right)}\right]^{C_{F} / b_{0}}, \\
Z_{m}\left(\mu^{2}\right) & =\left[\frac{\alpha_{s}\left(\mu^{2}\right)}{\alpha_{s}\left(\bar{M}_{Q}^{2}\right)}\right]^{3 C_{F} / b_{0}}, \\
Z_{m}^{k} & =Z_{m}\left(\mu^{2}=k^{2}\right), \\
Z_{m}^{\sigma} & =Z_{m}\left(\mu^{2}=\sigma^{2}\right),
\end{aligned}
$$

where $b_{0}=25 / 3$. The running $\overline{\mathrm{MS}}$ mass in Eqs. (84) is given by

$$
\bar{M}_{Q}\left(\mu^{2}\right)=Z_{m}\left(\mu^{2}\right) \bar{M}_{Q}
$$

Following $\mathrm{BC}$, we take the strong coupling $\alpha_{s}$ to be given approximately by

$$
\alpha_{s}\left(\mu^{2}\right) \approx \frac{4 \pi / b_{0}}{\log \left[\mu^{2} /(200 \mathrm{MeV})^{2}\right]} .
$$

In Eqs. (84), $\sigma^{2}$ is the square of the four-momentum of the charm-quark propagator in Fig. 1 of BC. Again, we follow BC and use the approximate expression

$$
\sigma^{2} \approx x q_{0}^{2}+\bar{M}_{Q}^{2}\left[1+\frac{1}{y(1-y)}\right] .
$$

The renormalization factors $Z_{i}$ in Eq. (84) were introduced in BC in order to account for the evolution of the heavy-quark mass and tensor and pseudoscalar currents from the scale $\bar{M}_{Q}$ to the scales of the hard interactions in the production process. We note that such renormalization factors do not appear in the NRQCD calculations of the cross section. As we shall see, they have a large numerical effect on the cross section. 
We use the following numerical values, which were given in $\mathrm{BC}: s=112 \mathrm{GeV}^{2}$, $f_{V}=f_{P}=400 \mathrm{MeV}, \bar{M}_{Q}=\bar{M}_{c}=1.2 \mathrm{GeV}$. We also take $M_{J / \psi}=3.096916 \mathrm{GeV}$ and $M_{\eta_{c}}=2.98040 \mathrm{GeV}$, which imply that $\bar{M}=3.06779 \mathrm{GeV}$. We note that, in the light-cone formalism, $f_{V}$ is related to the leptonic decay width of the $J / \psi$ as $\Gamma\left(J / \psi \rightarrow e^{+} e^{-}\right)=$ $\left(16 \pi \alpha^{2} / 27\right)\left|f_{V}\right|^{2} / M_{J / \psi}$. (See footnote 9 of BC.) Therefore, the value of $f_{V}$ is independent of the shape of light-cone distribution and, hence, is unaffected by the treatment of the high-momentum tail of the light-cone distribution. The value of $f_{P}$ is fixed by making use of the fact that $f_{P}$ is equal to $f_{V}$, up to corrections of relative order $v^{2}$, which are neglected.

\section{B. Numerical results}

Now we compute numerical values of the light-cone cross section (76), using the light-cone distribution amplitudes that we derived from the Cornell potential model.

As we have already mentioned, we drop contributions that arise from the light-cone distribution amplitude $V_{A}$, as these are of higher order in $v$. That is, we set the term $f_{5}$ in Eq. (80) to zero. For the model light-cone distribution amplitudes of BC, this has the effect of reducing the cross section from the value $\sigma\left(e^{+} e^{-} \rightarrow J / \psi+\eta_{c}\right) \approx 33 \mathrm{fb}$ reported in BC to $\sigma\left(e^{+} e^{-} \rightarrow J / \psi+\eta_{c}\right) \approx 23.47 \mathrm{fb}$. Thus, we do not expect the absence of this contribution to affect the order-of-magnitude discrepancy between the light-cone and NRQCD factorization results that we wish to address here.

The remaining light-cone distribution amplitudes are given in terms of the light-cone distribution amplitude that is derived from the potential-model wave function [Eq. (50)]. Hence, we take

$$
\begin{aligned}
& V_{T}(x)=V_{L}(x)=V_{\perp}(x)=\phi\left(x_{1}\right), \\
& P_{P}(y)=P_{A}(y)=\phi\left(y_{1}\right),
\end{aligned}
$$

where $\phi(z)$ is defined in Eq. (54), and we use the equivalent forms in Eqs. (59b) and (60) for the numerical evaluation. Substituting these light-cone distribution amplitudes into Eq. (80), we obtain the values for the cross section $\sigma\left(e^{+} e^{-} \rightarrow J / \psi+\eta_{c}\right)$ that are shown in the fourth row of Table II and are denoted by $\sigma_{0}$. If we correct the light-cone distribution amplitudes by subtracting the piece $\Delta \phi\left[\mathrm{Eq}\right.$. (73)] that corresponds to the order- $\alpha_{s}$ correction to the NRQCD short-distance coefficient, then we obtain the results that are shown in the fifth 
TABLE II: Values for the cross section $\sigma\left(e^{+} e^{-} \rightarrow J / \psi+\eta_{c}\right)$ computed using the light-cone distribution amplitudes that are derived from a potential-model wave function. As is explained in the text, $\sigma_{0}$ denotes the unsubtracted cross section, $\sigma$ denotes the cross section in which contributions that correspond to order- $\alpha_{s}$ correction to the NRQCD short-distance coefficient have been removed from the light-cone distribution amplitudes, and $\sigma_{\delta}$ denotes the cross section in which the light-cone distribution amplitudes have been replaced by $\delta$ functions. The notation "BC" indicates that the values of the renormalization constants $Z_{i}$ or $\alpha_{s}$ are those that were used in $\mathrm{BC}$. The value $\bar{M}_{c}=1.2 \mathrm{GeV}$ was used in BC; the values $\bar{M}_{c}=1.4 \mathrm{GeV}$ and $\alpha_{s}=0.21$ were used in Ref. [4]. The notation " $\overline{\mathrm{BC}}$ " indicates that, in Eq. (82), we have inserted two factors of $Z_{m}^{k}$ for each factor of $\bar{M}_{Q}^{2}$, as is described in the text below Eq. (82).

\begin{tabular}{c|ccccc}
\hline \hline$Z$ & $\mathrm{BC}$ & $\overline{\mathrm{BC}}$ & 1 & 1 & 1 \\
$\alpha_{s}$ & $\mathrm{BC}$ & $\mathrm{BC}$ & $\mathrm{BC}$ & 0.21 & 0.21 \\
$\bar{M}_{c}(\mathrm{GeV})$ & 1.2 & 1.2 & 1.2 & 1.2 & 1.4 \\
\hline$\sigma_{0}(\mathrm{fb})$ & 22.51 & 25.03 & 11.59 & 7.58 & 4.95 \\
$\sigma(\mathrm{fb})$ & 7.77 & 8.19 & 4.30 & 3.29 & 2.48 \\
$\sigma_{\delta}(\mathrm{fb})$ & 5.39 & 5.59 & 3.04 & 2.48 & 1.95 \\
\hline \hline
\end{tabular}

row of Table II and are denoted by $\sigma$. Finally, if we replace the light-cone distribution amplitudes $\phi(z)$ by $\delta(z-1 / 2)$, then we obtain the results that are shown in the sixth row Table II and are denoted by $\sigma_{\delta}$.

\section{Discussion}

As can be seen from Table II, if we take the values of $Z_{i}, \alpha_{s}$, and $\bar{M}_{c}$ that were used in BC, then the value of the unsubtracted cross section $\sigma$ that is obtained by using the potentialmodel light-cone distribution amplitude does not differ substantially from the value $23.47 \mathrm{fb}$ that is obtained by using the model light-cone distribution amplitudes of $\mathrm{BC}$ (with the contribution of $V_{A}$ set to zero). This is not surprising, since, as can be seen from Fig. 2, the potential-model light-cone distribution amplitude does not differ greatly in shape from the model light-cone distribution amplitudes of BC.

However, once the light-cone distribution amplitudes are corrected by subtracting the part 
$\Delta \phi$ that corresponds to the order- $\alpha_{s}$ correction to the NRQCD short-distance coefficient, then the cross section is reduced significantly - by a factor of approximately three if the values of $Z_{i}, \alpha_{s}$, and $\bar{M}_{c}$ are those that were used in BC.

A further large reduction in the cross section occurs if we set the renormalization factors $Z_{i}$ equal to unity. These factors represent an attempt to resum large logarithms of the heavy-quark virtuality in the production process divided by $\bar{M}_{c}^{2}$. However, it is not clear to us that these factors, as employed in $\mathrm{BC}$, correctly resum such logarithms. The factors $Z_{p}$ and $Z_{t}$ are inserted in an attempt to account for the evolution of the light-cone distributions from the scale $\bar{M}_{c}^{2}$ to the scale of the heavy-quark virtuality. However, these renormalization factors account correctly only for the evolution of the first moments of those distributions. The factor $Z_{m}$ evolves the heavy-quark mass in the heavy-quark propagator. However, some of the factors of $\bar{M}_{Q}$ in Eqs. (81) and (82) should not be evolved because they do not arise from the propagator mass, but, rather, from the approximation that the external heavyquark lines are taken to be on shell with mass $\bar{M}_{Q}$. Specifically, some of the factors of $\bar{M}_{Q}$ that appear in the quantities $d(x, y), s(x)$, and $s(y)$ [Eqs. (81) and (82)] arise in this way.

After we have set the renormalization factors $Z_{i}$ equal to unity, the cross section is reduced to $4.3 \mathrm{fb}$, which is comparable to the values $3.78 \pm 1.26 \mathrm{fb}$ (Ref. [4]) and $5.5 \mathrm{fb}$ (Ref. [5]) that were obtained in the NRQCD factorization calculations in leading order in $\alpha_{s}$. For purposes of comparison with the results of Ref. [4], we show in the last two columns of Table II the effects of taking the values of $\bar{M}_{c}$ and $\alpha_{s}$ that were used in that paper. It should be noted in making this comparison that the NLO value of the wave function at the origin that was used in Ref. [4] corresponds to $f_{V} \approx 489 \mathrm{MeV}$. Use of this value for $f_{V}$ (rather than $400 \mathrm{MeV}$ ) would enhance the cross section by about a factor 2.2 .

In $\mathrm{BC}$, it is claimed that a large enhancement of the cross section occurs in the lightcone calculation because it takes into account the finite width of the quarkonium wave function. However, we see from Table II that once the $\Delta \phi$ part of the light-cone distribution amplitudes has been subtracted, the cross section does not differ greatly from that which is obtained by using $\delta$-function light-cone distribution amplitudes. We conclude that any large enhancement that arises from the finite width of the quarkonium wave function must be coming from the region that corresponds to the order- $\alpha_{s}$ corrections to the NRQCD short-distance coefficient, i.e. the region of large heavy-quark momentum $\left(\gtrsim m_{c}\right)$. Within our potential-model calculation, it is essential to remove this region, as it is not treated 
reliably under the nonrelativistic approximations that go into that model. In comparing with NRQCD factorization calculations, it is necessary to identify the contribution from this region, even if one makes use of light-cone distribution amplitudes with the correct high-momentum behavior, because otherwise its contributions would be double counted in the NRQCD factorization corrections of order $\alpha_{s}$ to the production cross section. We note that the order- $\alpha_{s}$ corrections to the production cross section are indeed large [7].

The absence of a large enhancement of $\sigma$ relative to $\sigma_{\delta}$ is somewhat surprising, since it is known that, in the NRQCD factorization approach, there are large corrections of order $v^{2}$ (Ref. [4]) that account partially for the effects of the finite width of the quarkonium wave function. However, the order- $v^{2}$ corrections arise from several sources: the dependence of the amplitude on the $Q \bar{Q}$ relative momentum and the difference between the charmonium mass and $2 m_{c}$ in the phase space and in the relativistic normalizations of the states. Of these corrections, only the first type is sensitive to the finite width of the quarkonium wave function. It is $3.87\left\langle v^{2}\right\rangle$ relative to the lowest-order cross section [4]. Here, $\left\langle v^{2}\right\rangle=$ $\left\langle\mathcal{P}_{1}\right\rangle /\left(m_{c}^{2}\left\langle\mathcal{O}_{1}\right\rangle\right)$, where the matrix elements are taken in the $J / \psi$ or $\eta_{c}$ state, and the NRQCD operators $\mathcal{O}_{1}$ and $\mathcal{P}_{1}$ are defined in Ref. [6]. (Their matrix elements are proportional to the square of the wave function at the origin and the wave function at the origin times the second derivative of the wave function at the origin, respectively.) Taking $\left\langle\mathcal{P}_{1}\right\rangle /\left\langle\mathcal{O}_{1}\right\rangle=$ $0.50 \pm 0.09 \pm 0.15 \mathrm{GeV}^{2}$ (Ref. [19]) and $m_{c}=1.4 \mathrm{GeV}$, we see that the order $-v^{2}$ corrections that arise from the dependence of the amplitude on the $Q \bar{Q}$ relative momentum are approximately $99 \%$ of the lowest-order cross section. The difference between $\sigma$ and $\sigma_{\delta}$ is only approximately $29 \%$. However, it should be remembered that the light-cone formalism omits contributions that arise from the transverse part of the $Q \bar{Q}$ relative momentum. (Note, though, that the transverse-momentum contributions are suppressed by a factor $v^{2} M_{\text {meson }}^{2} / E_{\text {beam }}^{2}$ relative to the longitudinal-momentum contributions.) We also point out that, in dropping terms that are proportional to the light-cone distribution amplitude $V_{A}$, we have omitted corrections of order $v^{2}$ that, in the case of the model light-cone distributions of $\mathrm{BC}$, amount to about a $30 \%$ correction relative to the cross section computed without them. In order to be more quantitative about the effects of these omissions, we have calculated the order- $v^{2}$ correction that is associated with the finite widths of the light-cone distributions by expanding the light-cone cross section around $z=1 / 2$. The result is a relative correction $2.1\left\langle v^{2}\right\rangle \approx 54 \%$. Presumably, the remaining small difference between this correction and $\left(\sigma-\sigma_{\delta}\right) / \sigma$ of about 
$54 \%-29 \%=25 \%$ arises from corrections of still higher orders in $v$ and from the fact that we have computed $\delta \phi$ only at leading order in $\alpha_{s}$.

\section{SUMMARY}

The discrepancy between theoretical predictions in the NRQCD factorization approach and experimental measurements of the rate for the process $e^{+} e^{-} \rightarrow J / \psi+\eta_{c}$ at the $B$ factories presents a significant challenge to our understanding of the physics of quarkonia and, perhaps, to our understanding of QCD. In Ref. [8] (BC), it was suggested that the solution to this problem may lie in using the light-cone formalism to take into account the effects of the relative momenta of the heavy quarks and antiquarks in the quarkonia. The cross section calculated in $\mathrm{BC}$ is about a factor 6-10 times larger than the cross sections sections calculated at leading order in the NRQCD factorization approach $[4,5]$. Both the NRQCD factorization approach and the light-cone method are believed to approximate QCD in the limit in which the hard-scattering momentum transfer is much larger than the heavyquark mass or $\Lambda_{\mathrm{QCD}}$. Therefore, the apparent discrepancy between the two approaches presents a further, purely theoretical, puzzle. In this paper, we attempt to address this theoretical issue on several fronts.

First, we use a potential model to construct light-cone quarkonium distribution amplitudes that are a good approximation to the true light-cone distribution amplitudes of QCD. If one knows the static heavy-quark potential sufficiently accurately, then the potential-model allows one to determine the quarkonium wave function up to corrections of relative order $v^{2}$, where $v$ is the heavy-quark (or antiquark) velocity in the quarkonium rest frame. ( $v^{2} \approx 0.3$ for charmonium). We make use of the Cornell potential [17], which, for an appropriate choice of parameters, yields a good fit to the lattice data for the heavy-quark potential - thus giving us confidence in the connection of the potential model to QCD. We use the Bethe-Salpeter equation to relate the potential-model wave functions to the definitions of the light-cone distributions, which are written in terms of the Bethe-Salpeter wave function. Then, we use on-shell kinematics for the heavy quarks to relate momentum variables to light-cone variables. The latter approximation is valid up to corrections of relative order $v^{2}$. Finally, we integrate out the relative $Q \bar{Q}$ transverse momentum in the Bethe-Salpeter wave functions to form the light-cone distribution amplitudes. The light-cone distribution amplitudes that we 
derive agree reasonably well in shape with the model light-cone distribution amplitudes of $\mathrm{BC}$. The calculated cross section changes very little if we replace the light-cone distribution amplitudes of $\mathrm{BC}$ with ours. Thus, we conclude that the distribution amplitudes themselves are not the source of the theoretical discrepancy.

A part of the light-cone result for the production cross section arises from the region of large $Q \bar{Q}$ relative momentum ( $\left.\gtrsim m_{c}\right)$. This region is not treated accurately in our inherently nonrelativistic potential model. Furthermore, it consists of contributions that are part of the order- $\alpha_{s}$ correction to the hard-scattering coefficient in the NRQCD factorization approach. Hence, it is necessary to identify these contributions in order to avoid double-counting the order- $\alpha_{s}$ correction in making comparisons between the light-cone approach and the NRQCD factorization approach. After we subtract these contributions from the light-cone calculation, the production cross section is reduced by about a factor of three.

The light-cone cross section in BC contains renormalization factors $Z_{i}$ for the heavy-quark mass and the pseudo-scalar and tensor point-like vertices. If we set them to unity, a further reduction in the cross section of about a factor of two occurs. These renormalization factors have no counterpart in a conventional NRQCD factorization calculation. They represent an attempt to resum large logarithms of the heavy-quark virtuality in the production process divided by the square of the heavy-quark mass. However, as we have explained in Sec. VIC, we are not convinced that these renormalization factors, as employed in BC, resum such logarithms correctly. It is important to understand whether a correct resummation yields a significant enhancement to the cross section and, if so, to incorporate it into NRQCD factorization calculations, as well as into light-cone calculations.

Once the factors $Z_{i}$ have been set equal to unity, the subtracted light-cone cross section is comparable in size with the NRQCD factorization cross sections, and it differs by only about $29 \%$ from the light-cone cross section that is obtained by replacing the light-cone distribution amplitudes with zero-width distribution amplitudes ( $\delta$ functions).$^{5}$

We conclude that there is no conflict between the light-cone and NRQCD factorization

\footnotetext{
${ }^{5}$ Even in the case of $\delta$-function light-cone distribution amplitudes, there are small differences between the light-cone and NRQCD factorization cross sections. Some of these differences arise from different choices for the quarkonium wave functions at the origin, $\alpha_{s}$, and the charm-quark mass and from the inclusion of quantum-electrodynamic corrections in Ref. [4]. Other differences, presumably, are due to the fact that the light-cone and NRQCD factorization cross sections become equal only in the limit of infinite hard-scattering momentum transfer.
} 
results. Further, it seems that enhancements to the cross section that arise from making use of a light-cone wave function of finite width are rather modest and are not sufficient to remove the discrepancy between theory and experiment. That discrepancy remains an important puzzle. At this point, the most promising avenue for its resolution seems to be the inclusion of corrections of higher order in $\alpha_{s}$ (Ref.[7]) and $v$.

\section{Acknowledgments}

We wish to thank Eric Braaten for useful discussions. J.L. thanks the high energy theory group at Argonne National Laboratory for its hospitality. The research of G.T.B. in the High Energy Physics Division at Argonne National Laboratory is supported by the U. S. Department of Energy, Division of High Energy Physics, under Contract W-31-109-ENG-38. The work of J.L. is supported by the Korea Research Foundation under grant KRF-2004015-C00092. D.K.'s work is supported in part by the Seoul Science Fellowship and by the Korea Research Foundation under grant KRF-2006-612-C00003.

\section{APPENDIX A: LIGHT-CONE DISTRIBUTION AMPLITUDES FOR THE PURE COULOMB POTENTIAL AND ASYMPTOTIC BEHAVIOR AT $z=0,1$}

In this Appendix, we compute analytically the light-cone distribution amplitudes $\phi, \phi_{a}$, and $\phi_{b}$ for the special case of a pure Coulomb potential. We also determine their asymptotic behaviors at $z=0$ (or $z=1$ ). These analytic results are useful in checking the accuracy of the numerical-integration methods that we use in the more general case of the Cornell potential.

We drop the linear potential in the radial equation (6). In this pure Coulomb case, as is well known, Eq. (6) can be solved analytically. For the ground state, the wave function $u(\rho)$ and eigenvalue $\zeta$ are

$$
\begin{aligned}
u(\rho) & =\sqrt{\frac{\lambda^{3}}{2}} \rho e^{-\frac{\lambda}{2} \rho}, \\
\zeta_{10} & =-\frac{\lambda^{2}}{4}
\end{aligned}
$$


Applying the relations in Eqs. (3), (5a), (5b), and (7), we obtain

$$
\begin{aligned}
R(r) & =2 \gamma_{\mathrm{C}}^{3 / 2} e^{-\gamma_{\mathrm{C}} r}, \\
\epsilon_{\mathrm{B}} & =-\frac{\gamma_{\mathrm{C}}^{2}}{m} .
\end{aligned}
$$

From Eq. (A3a), it follows that

$$
[\psi(r)]_{\text {Coulomb }}=\psi(0) e^{-\gamma_{\mathrm{C}} r},
$$

where the wave function at the origin is given by

$$
|\psi(0)|=\sqrt{\frac{\gamma_{\mathrm{C}}^{3}}{\pi}} .
$$

We next use Eq. (A4) to calculate the light-cone distribution amplitude in the pure Coulomb case. Substituting Eq. (A4) into Eq. (59) and integrating over $r$, we obtain

$$
\begin{aligned}
{[\phi(z)]_{\text {Coulomb }} } & =\frac{4 \gamma_{\mathrm{C}}}{\pi} \int_{\sqrt{d(z)}}^{\infty} \frac{|\boldsymbol{p}| \sqrt{|\boldsymbol{p}|^{2}+m_{c}^{2}}}{\left(|\boldsymbol{p}|^{2}+\gamma_{\mathrm{C}}^{2}\right)^{2}} d|\boldsymbol{p}|, \\
{\left[\phi_{a}(z)\right]_{\text {Coulomb }} } & =\frac{4 \gamma_{\mathrm{C}}}{\pi} \int_{\sqrt{d(z)}}^{\infty} \frac{|\boldsymbol{p}|^{2}}{\left(|\boldsymbol{p}|^{2}+\gamma_{\mathrm{C}}^{2}\right)^{2}} d|\boldsymbol{p}|,
\end{aligned}
$$

where we have used $\phi=\phi_{a}+\phi_{b}$. Carrying out the integration over $|\boldsymbol{p}|$ in Eqs. (A6), we have

$$
\begin{aligned}
{[\phi(z)]_{\text {Coulomb }} } & =\frac{2 \gamma_{\mathrm{C}}}{\pi}\left[\frac{\sqrt{d(z)+m_{c}^{2}}}{d(z)+\gamma_{\mathrm{C}}^{2}}+\frac{\sinh ^{-1} \sqrt{\frac{m_{c}^{2}-\gamma_{\mathrm{C}}^{2}}{d(z)+\gamma_{\mathrm{C}}^{2}}}}{\sqrt{m_{c}^{2}-\gamma_{\mathrm{C}}^{2}}}\right], \\
{\left[\phi_{a}(z)\right]_{\text {Coulomb }} } & =\frac{2}{\pi}\left[\frac{\gamma_{\mathrm{C}} \sqrt{d(z)}}{d(z)+\gamma_{\mathrm{C}}^{2}}+\arctan \left(\frac{\gamma_{\mathrm{C}}}{\sqrt{d(z)}}\right)\right] .
\end{aligned}
$$

Note that Eq. (A7b) can also be obtained by substituting the wave function in Eq. (A4) into Eq. (60) and integrating over $r$.

We can use our results in the pure Coulomb case to compute the asymptotic behavior of the light-cone distribution amplitudes at $z=0$ (or $z=1$ ). At $z=0$, the corresponding longitudinal momentum is infinite [Eq. (51a)], and only the short-distance behavior of $\psi(r)$ is important. That short-distance behavior is governed by the Coulomb potential. ${ }^{6}$ Taking

\footnotetext{
${ }^{6}$ By differentiating Eqs. (59a) and (59b) with respect to $z$ and setting $z=0$, one can verify that, at $z=0$, only the large $p$ behaviors of the integrands for $\phi_{a}$ and $\phi_{b}$ are important and, hence, that only the short-distance behavior of $\psi(r)$ is important. This approach yields a simple expression for the asymptotic behaviors of the derivatives of the light-cone distribution amplitudes with respect to $z$, which can then be translated into the asymptotic behaviors of the distribution amplitude.
} 
the limit $z \rightarrow 0$ in Eqs. (A7a) and (A7b) and using $\phi_{b}=\phi-\phi_{a}$, we find the asymptotic behaviors

$$
\begin{aligned}
\phi(z) & \sim \phi_{a}(z) \sim \frac{8 \gamma_{C}}{\pi m_{c}} z^{1 / 2} \\
\phi_{b}(z) & \sim \frac{16 \gamma_{C}}{3 \pi m_{c}} z^{3 / 2}
\end{aligned}
$$

The asymptotic forms in Eqs. (A8) provide good tests of the convergence in the highmomentum region of the numerical integrations that we use to compute $\phi(z)$ and $\phi_{a}(z)$.

[1] P. Pakhlov [Belle Collaboration], arXiv:hep-ex/0412041.

[2] K. Abe et al. [Belle Collaboration], Phys. Rev. D 70, 071102 (2004) [arXiv:hep-ex/0407009].

[3] B. Aubert et al. [BABAR Collaboration], Phys. Rev. D 72, 031101 (2005) [arXiv:hepex/0506062].

[4] E. Braaten and J. Lee, Phys. Rev. D 67, 054007 (2003) [Erratum-ibid. D 72, 099901 (2005)] [arXiv:hep-ph/0211085].

[5] K. Y. Liu, Z. G. He, and K. T. Chao, Phys. Lett. B 557, 45 (2003) [arXiv:hep-ph/0211181].

[6] G. T. Bodwin, E. Braaten, and G. P. Lepage, Phys. Rev. D 51, 1125 (1995) [Erratum-ibid. D 55, 5853 (1997)] [arXiv:hep-ph/9407339].

[7] Y. J. Zhang, Y. j. Gao, and K. T. Chao, Phys. Rev. Lett. 96, 092001 (2006) [arXiv:hep$\mathrm{ph} / 0506076]$.

[8] A. E. Bondar and V. L. Chernyak, Phys. Lett. B 612, 215 (2005) [arXiv:hep-ph/0412335].

[9] J. P. Ma and Z. G. Si, Phys. Rev. D 70, 074007 (2004) [arXiv:hep-ph/0405111].

[10] V. V. Braguta, A. K. Likhoded, and A. V. Luchinsky, Phys. Rev. D 72, 074019 (2005) [arXiv:hep-ph/0507275].

[11] V. V. Braguta, A. K. Likhoded, and A. V. Luchinsky, Phys. Lett. B 635, 299 (2006) [arXiv:hep-ph/0602047].

[12] G. C. Nayak, J. W. Qiu, and G. Sterman, Phys. Lett. B 613, 45 (2005) [arXiv:hep$\mathrm{ph} / 0501235]$.

[13] G. C. Nayak, J. W. Qiu, and G. Sterman, Phys. Rev. D 72, 114012 (2005) [arXiv:hepph/0509021].

[14] G. T. Bodwin, Int. J. Mod. Phys. A 21, 785 (2006) [arXiv:hep-ph/0509203]. 
[15] G. C. Nayak, J. W. Qiu and G. Sterman, Phys. Rev. D 74, 074007 (2006) [arXiv:hep$\mathrm{ph} / 0608066]$.

[16] N. Brambilla, A. Pineda, J. Soto, and A. Vairo, Nucl. Phys. B 566, 275 (2000) [arXiv:hep$\mathrm{ph} / 9907240]$.

[17] E. Eichten, K. Gottfried, T. Kinoshita, K. D. Lane, and T. M. Yan, Phys. Rev. D 17, 3090 (1978) [Erratum-ibid. D 21, 313 (1980)].

[18] G. S. Bali, Phys. Rept. 343, 1 (2001) [arXiv:hep-ph/0001312].

[19] G. T. Bodwin, D. Kang, and J. Lee, Phys. Rev. D 74, 014014 (2006) [arXiv:hep-ph/0603186].

[20] S. P. Booth, D. S. Henty, A. Hulsebos, A. C. Irving, C. Michael, and P. W. Stephenson [UKQCD Collaboration], Phys. Lett. B 294, 385 (1992) [arXiv:hep-lat/9209008].

[21] R. Gupta and T. Bhattacharya, Phys. Rev. D 55, 7203 (1997) [arXiv:hep-lat/9605039].

[22] S. Kim and D. K. Sinclair, Phys. Rev. D 48, 4408 (1993).

[23] S. Kim and S. Ohta, Nucl. Phys. Proc. Suppl. 53, 199 (1997) [arXiv:hep-lat/9609023].

[24] V. L. Chernyak and A. R. Zhitnitsky, Phys. Rept. 112, 173 (1984).

[25] J. H. Kühn, J. Kaplan, and E. G. Safiani, Nucl. Phys. B157, 125 (1979).

[26] B. Guberina, J. H. Kühn, R. D. Peccei, and R. Rückl, Nucl. Phys. B174, 317 (1980).

[27] G. T. Bodwin and A. Petrelli, Phys. Rev. D 66, 094011 (2002) [arXiv:hep-ph/0205210].

[28] E. E. Salpeter and H. A. Bethe, Phys. Rev. 84, 1232 (1951).

[29] E. E. Salpeter, Phys. Rev. 87, 328 (1952).

[30] M. Beneke and V. A. Smirnov, Nucl. Phys. B 522, 321 (1998) [arXiv:hep-ph/9711391].

[31] S. J. Brodsky and G. P. Lepage, Phys. Rev. D 24, 2848 (1981). 